\title{
Lateral Casimir force between sinusoidally corrugated surfaces: Asymmetric profiles, deviations from the proximity force approximation and comparison with exact theory
}

\author{
H.-C. Chiu, ${ }^{1}$ G. L. Klimchitskaya, ${ }^{2, *}$ V. N. Marachevsky, ${ }^{3,+}$ \\ V. M. Mostepanenko,,+ and U. Mohideen ${ }^{1, \S}$ \\ ${ }^{1}$ Department of Physics and Astronomy, \\ University of California, Riverside, California 92521, USA \\ ${ }^{2}$ North-West Technical University, Millionnaya St. 5, St.Petersburg, 191065, Russia \\ ${ }^{3}$ V.A. Fock Institute of Physics, Saint-Petersburg \\ State University, St.Petersburg, 198504, Russia \\ ${ }^{4}$ Noncommercial Partnership "Scientific Instruments", \\ Tverskaya St. 11, Moscow, 103905, Russia
}




\begin{abstract}
The lateral Casimir force, which arises between aligned sinusoidally corrugated surfaces of a sphere and a plate, was measured for the case of a small corrugation period beyond the applicability region of the proximity force approximation. The increased amplitudes of the corrugations on both the sphere and the plate allowed observation of an asymmetry of the lateral Casimir force, i.e., deviation of its profile from a perfect sine function. The dependences of the lateral force on the phase shift between the corrugations on both test bodies were measured at different separations in two sets of measurements with different amplitudes of corrugations on the sphere. The maximum magnitude of the lateral force as a function of separation was also measured in two successive experiments. All measurement data were compared with the theoretical approach using the proximity force approximation and with the exact theory based on Rayleigh expansions with no fitting parameters. In both cases real material properties of the test bodies and nonzero temperature were taken into account. The data were found to be in a good agreement with the exact theory but deviate significantly from the predictions of the proximity force approximation approach. This provides the quantitative confirmation for the observation of diffraction-type effects that are disregarded within the PFA approach. Possible applications of the phenomenon of the lateral Casimir force in nanotechnology for the operation of micromachines are discussed.
\end{abstract}

PACS numbers: 78.20.Ci, 68.35.Af, 68.35.Ct, 85.85.+j

*Electronic address: Galina.Klimchitskaya@itp.uni-leipzig.de

${ }^{\dagger}$ Electronic address: maraval@mail.ru

‡Electronic address: Vladimir.Mostepanenko@itp.uni-leipzig.de

§Electronic address: Umar.Mohideen@ucr.edu 


\section{INTRODUCTION}

The Casimir effect [1] is presently well known due to the many potential applications in both fundamental physics and nanotechnology. The Casimir force is an extension of the van der Waals interaction to larger separations between macroscopic bodies where the relativistic retardation of the electromagnetic interaction becomes important due to the finite speed of light. The Casimir effect originates from the existence of zero-point and thermal oscillations in restricted quantization volumes. The results of extensive experimental and theoretical studies on the role of the Casimir force in configurations with idealized boundary surfaces (infinitely thin, with ideal-metal boundary conditions etc.) and also between surfaces of real material bodies with account of roughness, nonzero skin depth and nonzero temperature are presented in Ref. [2] (see also earlier books and reviews [3-10]). Interrelationship between experiment and theory in the case of real materials was specially reviewed in Ref. [11]. In condensed matter physics the Casimir force is taken into account in the investigation of various properties of thin films, surface tension, in atomic force microscopy and critical phenomena [12]. When the characteristic sizes of microdevices shrink below a micrometer, the role of the Casimir and electric forces become comparable. This opens new opportunities for the creation of nanoscale devices actuated by the Casimir force [13, 14]. Investigation of the combined action of the electric and Casimir forces in microdevices is vital for the resolution of issues associated with their stability [15, 16].

The most universally known normal Casimir force acts in the direction perpendicular to the interacting surfaces. However, when the material properties of the interacting bodies are anisotropic or they are asymmetrically positioned, a lateral Casimir force may exist which acts tangential to the surface. Similar to the normal Casimir force, the lateral force originates from the modification of electromagnetic zero-point and thermal oscillations by material boundaries. For plates made of anisotropic materials, the lateral Casimir force and related torques were theoretically predicted in Refs. [17, 18] (see also Refs. [4, 19]). For two parallel ideal metal plates covered with uniaxial sinusoidal corrugations of equal periods, the lateral Casimir force was predicted[20, 21] and calculated [22, 23] in the second perturbation order with respect to the amplitudes of corrugations. In both cases a sinusoidal dependence of the result on the respective characteristic angle was obtained. For sinusoidally corrugated plates made of real metal described by the plasma model, the lateral Casimir 
force was considered in Refs. [24, 25] in second order perturbation theory. In the case of two ideal metal parallel plates covered with periodic uniaxial corrugations of rectangular shape, some results for the normal Casimir force were found in Refs. [26, 27]. It is pertinent to note that the use of normal and lateral Casimir forces gives the possibility to actuate both normal and lateral translations of corrugated surfaces in micromachines by means of the electromagnetic zero-point oscillations. The lateral Casimir force might be used to solve the tribological problems plaguing the microdevice industry. Specifically, it was suggested [2830] to use this effect for frictionless transmission of lateral motion. There are also proposals [31, 32] to measure the Casimir torque arising between the anisotropic bodies due to the lateral Casimir force.

Both the normal and lateral Casimir forces are in general nonadditive and possess a complicated dependence on the geometrical shape of the boundary surfaces connected with diffraction effects. In the configuration of a smooth $\mathrm{Al}$ coated sphere above a sinusoidally corrugated $\mathrm{Al}$ coated plate the nontrivial behavior of the normal Casimir force was experimentally demonstrated [33]. This was done in an additive regime when the sphere-plate separation is much smaller than the period of corrugations [23]. Recently [34] the nontrivial behavior of the normal Casimir force caused by the complicated geometry of a boundary surface was observed in the configuration of a smooth Au coated sphere above a Si plate covered with rectangular corrugations (trenches). In this case the nonadditive regime was used where separation distances are of the order of corrugation period. The deviations of the measurement data from the additive theory were reported [34]. These deviations are, however, about $50 \%$ less than those expected for ideal metal boundaries in the theory taking exact account of the geometrical shape [27]. The remaining discrepancy might be explained by the role of a nonzero skin depth which was not accounted for in Ref. [27]. Thus, the scattering approach for grating geometries taking the exact account of surface geometry at zero temperature and describing metallic properties by means of the simple plasma model [35, 36] was found to be in better agreement with the data of Ref. [34].

The first measurement of the lateral Casimir force was performed between the surfaces of an Au coated sphere and an Au coated plate both covered with aligned uniaxial corrugations of sinusoidal shape [37, 38]. The corrugation amplitudes were $A_{1}=59 \mathrm{~nm}$ on the plate and $A_{2}=8 \mathrm{~nm}$ on the sphere. The period of corrugations was equal to $1200 \mathrm{~nm}$, i.e., much larger than separation distances between the sphere and plate. This means that the experiments of 
Refs. [37, 38] was done in the application region of the proximity force approximation (PFA) [2, 8, 11]. The lateral Casimir force with an amplitude of $3.2 \times 10^{-13} \mathrm{~N}$ at the shortest separation of $221 \mathrm{~nm}$ was found to sinusoidally oscillate as a function of the phase shift between the corrugations. The total experimental error of the force amplitude measurement at the closest point was determined to be around $19 \%$ at the $95 \%$ confidence level [2]. The experimental results were compared with theory using the PFA which should supposedly be applicable at separations much smaller than the corrugation period. In so doing the dielectric properties of $\mathrm{Au}$ were described using the simple plasma model in fourth order perturbation theory with respect to a small parameter related to the ratio of the skin depth to the separation distance. The experimental results were found to be in good agreement with the theory in the limits of the experimental error. It was also predicted theoretically (but not observed experimentally due to the use of small corrugation amplitudes and insufficient precision) that the lateral Casimir force is asymmetric, i.e., deviates from purely sinusoidal harmonic dependence on the phase shift, through the contribution of higher order terms in the perturbation expansion with respect to the corrugation amplitudes.

In this paper we present complete measurement data of the recently performed experiment on the lateral Casimir force between a sinusoidally corrugated sphere and plate covered with Au layers. The much smaller period of corrugations used in this experiment provides opportunities to measure deviations of the lateral Casimir force from the theoretical predictions obtained on the basis of the PFA. Much deeper corrugations on both the sphere and the plate and shorter separations where the measurements were performed make it possible to demonstrate the asymmetry of the lateral Casimir force, as predicted in Ref. [38]. The measurement data are compared with the exact theory describing the Rayleigh scattering of the electromagnetic oscillations on the sinusoidally corrugated boundary surfaces of a sphere and a plate with no fitting parameters. Only the configuration of a perfect sphere near a plane plate is treated using the PFA. At the separations considered, this leads to only a negligibly small error of order $0.1 \%[2,11]$. The dielectric properties of Au are described by means of the generalized plasma-like model [39, 40] which takes into account the interband transitions of core electrons. The computational results for the amplitude of the lateral Casimir force as a function of separation and for the lateral Casimir force as a function of the phase shift at different separations obtained using the exact theory at the laboratory temperature $(T=300 \mathrm{~K})$ are found to be in a very good agreement with the measurement 
data. The same data deviate markedly from the theoretical results computed using the PFA. This clearly demonstrates the influence of diffraction effects on the lateral Casimir force.

Some of the results of this paper related to the first set of our measurements were briefly published in Ref. [41]. Here, we present additional phase dependences of the lateral Casimir force not included in Ref. [41] for the same corrugation amplitudes, as were used in [41], and also the results of further experiments performed with a much larger corrugation amplitude on the sphere. We demonstrate that the experimental data of various measurements for different samples are in mutual agreement. We also present additional important evidence characterizing the quality of corrugated surfaces, procedures of electrostatic calibrations, and details of error analysis. Calculations using the PFA are done by means of the exact Lifshitz formula rather than with the help of perturbation theory in corrugation amplitudes, as in Ref. [41]. The role of surface roughness is estimated. The details of exact computations of the lateral Casimir force using the Rayleigh scattering theory at nonzero temperature given in this paper have never been presented previously.

The paper is organized as follows. In Sec. II we describe the experimental setup and sample preparation. Section III contains the description of the procedures of electrostatic calibration using both the normal and lateral electric forces. In Sec. IV the measurement data and the error analysis are presented. Section $\mathrm{V}$ is devoted to the computation of the lateral Casimir force between corrugated surfaces using the PFA. In Sec. VI the lateral Casimir force is computed in a more fundamental way using the Rayleigh theory. In both Secs. V and VI the theoretical results are compared with the experimental data. Section VII contains our conclusions and discussion.

\section{EXPERIMENTAL SETUP AND SAMPLE PREPARATION}

A schematic diagram of the experimental setup is shown in Fig. 1, The experiments under discussion are performed using a standard atomic force microscope (AFM) in oil-free vacuum chamber at a pressure below 10 mTorr and at room temperature. The observation of the lateral Casimir force is done using two test bodies whose surfaces are covered with longitudinal sinusoidal corrugations of the same period. The axes of the corrugations should be perfectly parallel. Following [38], one can see that misalignment by $1^{\circ}$ of the axes of corrugations can lead to the loss of any lateral force. As the first test body, we have used 
a sinusoidally corrugated grating of size $5 \times 5 \mathrm{~mm}^{2}$ vertically mounted on the piezotube of an AFM (see Fig. 1). Here, the corrugations have an average period $\Lambda=574.7 \mathrm{~nm}$ (i.e., more than two times smaller than in Refs. [37, 38] in order to achieve the regime where the PFA becomes inappropriate for the calculation of the lateral Casimir force between the corrugated surfaces). The amplitude of corrugations on the grating $A_{1}=85.4 \pm 0.3 \mathrm{~nm}$ was a factor of 1.45 larger than in Refs. [37, 38]. A $320 \mu \mathrm{m}$ long V-shaped silicon nitride cantilever of the AFM was first uniformly coated with $40 \mathrm{~nm}$ of Al. This modification of cantilever was performed to improve its thermal and electric conductivity and prevent deformation due to differential thermal expansion in vacuum.

A polystyrene sphere of a $200 \pm 4 \mu \mathrm{m}$ diameter was placed at the end of the cantilever with conductive Ag epoxy. Next, a freshly cleaved mica sheet of $400 \mu \mathrm{m}$ length, $200 \mu \mathrm{m}$ width, and a few micrometer thickness was attached to the bottom of the sphere also with Ag epoxy. Then a second polystyrene sphere with a nominal diameter $2 R=200 \pm 4 \mu \mathrm{m}$ was attached to the bottom free end of the mica sheet (see Fig. 1). After imprint of corrugations on its surface (see below), this last sphere was used as the second test body in the measurement of the lateral Casimir force. We emphasize that the silver epoxy is rigid at all attachments. The first sphere and mica sheet are needed to isolate the laser reflection spot on the cantilever tip from the interaction region between the two corrugated surfaces. As a result, the effect of scattered light from the top and sides of the corrugated plate is substantially reduced. The resulting system (cantilever, mica sheet and the attached two spheres) was uniformly coated with a $10 \mathrm{~nm}$ layer of $\mathrm{Cr}$ and then with a more than $50 \mathrm{~nm}$ layer of $\mathrm{Au}$ in a thermal evaporator. Uniformity of coating was ensured through rotation of the cantilever [42]. The Cr layer was used to improve the adhesion of the Au coating and prevent its peeling during the imprint of the corrugations on the sphere.

The lateral Casimir force arises when the uniaxial corrugations on the two test bodies are perfectly aligned and have the same period $\Lambda$ [20 23, 37, 38]. To ensure that these conditions are satisfied, we have imprinted corrugations on the second sphere using the grating as a template. In order to obtain deeper corrugations than in Refs. [37, 38], a more sophisticated procedure was implemented. Here, the template grating had a $300 \mathrm{~nm}$ $\mathrm{Au}$ coating applied by the manufacturer on top of sinusoidal corrugations made of hard epoxy on a 3-mm-thick Pyrex substrate. Note that hard epoxy does not require the use of the oxide of Al layer which was coated on the soft plastic grating in Refs. [37, 38]. The 
smaller period of corrugations used here required the use of a precise stepper motor and piezocontrolled imprinting technique. The imprinting procedure was done as follows. At first, the second sphere was brought in contact with the grating using a micromanipulator. Then a hard flat stylus (see Fig. 1) was moved in $10 \mu \mathrm{m}$ steps using a stepper motor until it comes into contact with the other side of the sphere which is now sandwiched against the grating. Next the imprinting was done by applying a voltage to the $z$ piezo to gently press the sphere between the grating and the hard flat stylus. To obtain deeper imprints while preserving sphericity some additional pressure was put on the sphere using the stepper motor. Special care was needed at this step. If the applied pressure from the hard flat stylus was too much, the resulting rotation of the second sphere will cause the cantilever to break. There is less concern on the preservation of sphericity as polystyrene spheres are elastic. Then the voltage on the $z$-piezo is reversed to remove the pressure on the sphere. With the help of the same stepper motor the hard flat stylus was gently removed and the sphere was then translated horizontally by around $200 \mu \mathrm{m}$ to a different position on the grating. The last operation is necessary because the part of the grating used to imprint corrugations on the sphere might have changed its local amplitude during the imprinting process. This translation was performed preserving the orientation of the cantilever. The same imprinting procedure, but with application of larger pressure by means of the stepper motor, was done with a similar sphere in the second set of our measurements. After the imprint process was done, the system was left in vacuum for more than 2 hours to reach a stable equilibrium.

The corrugations on the grating and on both spheres were examined using an AFM and found to be rather homogeneous. This was done after the completion of the experiment. An AFM scan of the Au-coated corrugations of the vertical template grating is shown in Fig. 2(a). The value of the amplitude of these corrugations $A_{1}$, also measured using the AFM, is indicated above. The variance of the stochastic roughness on the grating was estimated in the following way. The topography of the groove from an AFM scan [see Fig. 2(a)] was fitted to a sine function. Note that the top and bottom of corrugations are symmetric as expected for a sine. This is illustrated in Fig. 2(b) where a typical section of Fig. 2(a) along a $y=$ const plane is shown. Then the difference of heights between the groove topography and the sine function was calculated at a sufficient number of intermediate points. The variance was found using these differences as the root-mean-square deviation between the two curves. A total 30 grooves were used to evaluate the average value of the variance 
leading to $\delta_{1}=13 \mathrm{~nm}$. In Fig. 3(a) we show an AFM scan of the corrugations imprinted on the sphere used in the first set of our measurements. The amplitude of these corrugations over a $30 \times 30 \mu \mathrm{m}^{2}$ area on the sphere was measured with an AFM to be $A_{2}=13.7 \pm 0.4 \mathrm{~nm}$ (i.e., an increase by a factor of 1.7 as compared with Refs. [37, 38]). Figure [3(b) is a typical section of Fig. 3(a) along a $y=$ const plane. It illustrates a symmetry between the top and bottom of the imprinted corrugations. The variance of the stochastic roughness of the imprinted grooves $\delta_{2}$ was found using the same procedure as for a grating with the result $\delta_{2}=2.7 \mathrm{~nm}$.

In Fig. 4 the AFM scan of a $5 \times 5 \mu \mathrm{m}^{2}$ area of imprinted corrugations on the sphere used in the first set of measurements is demonstrated. Here, the lighter tone shows higher points, as indicated in the additional scale. As is seen in Fig. 4, the imprinting procedure did not destroy the sphericity of the imprinted surface. An AFM scan of the sphere surface with imprinted corrugations used in the second set of our measurements was also performed. The amplitude of these corrugations was measured to be $\tilde{A}_{2}=25.5 \pm 0.6 \mathrm{~nm}$ (increase by a factor of 3.2 as compared with Refs. [37, 38]). The respective variance of the stochastic roughness in this case was $\tilde{\delta}_{2}=8.8 \mathrm{~nm}$. Note that the relatively large values of the variance of the stochastic roughness presented above (in comparison with the case of flat surfaces [43]) are explained by the fact that the r.m.s. deviation is calculated along the $z$-axis, i.e., not perpendicular to the corrugated surface at most of the intermediate points. In Fig. 5 we show a $5 \times 5 \mu \mathrm{m}^{2}$ area of the corrugations imprinted on the sphere used in the second set of our measurements. As can be seen in this figure, the grooves are deeper than for the sphere used in the first set of measurements, and the sphericity of the surface is preserved. The diameters of the spheres with the imprinted corrugations used in the first and second set of measurements were measured to be $2 R=194.0 \pm 0.3 \mu \mathrm{m}$ and $2 \tilde{R}=194.8 \pm 0.3 \mu \mathrm{m}$, respectively, using a scanning electron microscope (SEM). The SEM was calibrated with a NIST-traceable calibration grating which was independently cross calibrated with our interferometrically calibrated AFM.

The distinctive feature of our setup is that the lateral Casimir force acting along the $x$-axis tangential to the corrugated sphere and a grating leads to the vertical bending of the cantilever. This bending is measured using bicell photodiodes A and B in Fig. 1. Whereas a force acting normal to the test bodies (the normal Casimir force) leads to a torsional deflection of the cantilever. The torsional spring constant of the used cantilever $K_{\text {tor }}$ was 
found to be 46 times larger than the bending spring constant $K_{\text {ben }}$ (see the next section). Because of this the normal Casimir force could lead to only a negligible change in the position of the second sphere and in the phase of the corrugations. Thus the setup used is much more sensitive to detecting the lateral Casimir force, while simultaneously suppressing the effect of the normal Casimir force.

\section{ELECTROSTATIC CALIBRATIONS}

The calibration of the cantilever (i.e., the determination of its spring constants), the measurement of the residual potential difference $V_{0}$ between the sphere and the plate, and the determination of the separation on contact $z_{0}$ were done by using both the normal and lateral electric forces. We begin with the determination of $K_{\text {tor }}$ and $V_{0}$ by means of the normal electric force. For this purpose we have measured the torsional cantilever deflection due to the voltages $V$ applied to the grating while the sphere remained grounded. This was performed through the measurement of the difference signal between the bicell photodiodes shown in Fig. 1. To determine the parameters of our interest, the measured deflection signal $S_{\text {nor }}^{\text {el }}$ at some separation $a$ between the zero levels of corrugations on the grating and on the sphere, where the Casimir force is negligible, should be fitted to the theoretical expression for the normal electrostatic force between the corrugated surfaces. To attain these ends we begin with a brief derivation of such an expression in the sphere-plate configuration which is not available in the literature.

In the configuration of an ideal metal sphere above an ideal metal plate with smooth boundary surfaces the exact expression for the electric force is well known [44]. In the range of separations from $a=100 \mathrm{~nm}$ to $a=6 \mu \mathrm{m}$ it can be represented [45] by the following polynomial with a relative error less than $10^{-4 \%}$

$$
\begin{aligned}
& F_{\text {nor }}^{\mathrm{el}, \mathrm{sp}}(a)=-2 \pi \epsilon_{0}\left(V-V_{0}\right)^{2} \Phi(a), \\
& \Phi(a)=\sum_{i=-1}^{6} c_{i}\left(\frac{a}{R}\right)^{i},
\end{aligned}
$$

where $\epsilon_{0}$ is the permittivity of free space and the numerical coefficients $c_{i}$ are given by

$$
\begin{aligned}
& c_{-1}=0.5, \quad c_{0}=-1.18260, \quad c_{1}=22.2375, \quad c_{2}=-571.366, \\
& c_{3}=9592.45, \quad c_{4}=-90200.5, \quad c_{5}=383084 ., \quad c_{6}=-300357 .
\end{aligned}
$$


The approximate expression for the electric force in the case when the surfaces of a sphere and a plate are covered with sinusoidal corrugations can be obtained using the PFA, as applied to electric forces. For this purpose we consider the separation along the $z$-axis between any two points $z_{s}$ on the corrugated sphere and $z_{p}$ on the corrugated plate

$$
z_{s}-z_{p}=a+A_{2} \sin (2 \pi x / \Lambda+\varphi)-A_{1} \sin (2 \pi x / \Lambda) .
$$

Here $a$, as defined above, is the closest separation between a perfectly shaped sphere and a plate, and $\varphi \equiv 2 \pi x_{0} / \Lambda$ is the phase shift between the corrugations of both bodies. Then, according to the PFA, the normal electric force between corrugated a sphere and a plate is obtained from Eq. (1) by averaging over a period as

$$
F_{\text {nor }}^{\mathrm{el}}(a, \varphi)=-2 \pi \epsilon_{0}\left(V-V_{0}\right)^{2} \frac{1}{\Lambda} \int_{0}^{\Lambda} d x \Phi\left(z_{s}-z_{p}\right) .
$$

The integral in (4) can be calculated by using the following substitution of (3):

$$
z_{s}-z_{p}=a[1+\beta \cos (2 \pi x / \Lambda-\alpha)]
$$

where

$$
\begin{aligned}
& \beta \equiv \beta(a, \varphi)=\frac{1}{a}\left(A_{1}^{2}+A_{2}^{2}-2 A_{1} A_{2} \cos \varphi\right)^{1 / 2} \\
& \tan \alpha=\left(A_{2} \cos \varphi-A_{1}\right) /\left(A_{1} \sin \varphi\right) .
\end{aligned}
$$

Substituting Eq. (5) into Eq. (41) and performing integration, one obtains

$$
\begin{aligned}
& F_{\text {nor }}^{\mathrm{el}}(a, \varphi)=-2 \pi \epsilon_{0}\left(V-V_{0}\right)^{2}\left[\frac{R}{2 a} \frac{1}{\sqrt{1-\beta^{2}}}\right. \\
& +c_{0}+c_{1} \frac{a}{R}+c_{2} \frac{a^{2}\left(2+\beta^{2}\right)}{2 R^{2}}+c_{3} \frac{a^{3}\left(2+3 \beta^{2}\right)}{2 R^{3}} \\
& +c_{4} \frac{a^{4}\left(8+24 \beta^{2}+3 \beta^{4}\right)}{8 R^{4}}+c_{5} \frac{a^{5}\left(8+40 \beta^{2}+15 \beta^{4}\right)}{8 R^{5}} \\
& \left.+c_{6} \frac{a^{6}\left(16+120 \beta^{2}+90 \beta^{4}+5 \beta^{6}\right)}{16 R^{6}}\right] .
\end{aligned}
$$

It is well known that for corrugated surfaces the use of the PFA may not lead to precise expressions for the Casimir force [23 25]. For a static electric force, however, the PFA should work much better because in this case the diffraction effects are absent. To verify the validity of Eq. (7), we applied a 3-dimensional finite element analysis (FEA) using Comsol Multiphysics [46] to numerically solve the Poisson equation with appropriate boundary 
conditions in the configuration of corrugated sphere and plate. In the FEA computation, it is difficult to use the whole corrugated sphere and plate due to computer limitations. This necessitates the use of truncated spheres and parts of the plates. Such a replacement is justified as the primary contribution to the force comes from sphere-plate regions which are in close proximity. However, the error introduced by the truncation has to be independently confirmed to be negligible by varying the size of the regions of the sphere and plate used in the computations.

Computations were performed as a function of the common size $l$ of a truncated section of the corrugated sphere and a square corrugated plate. The separation distance and phase difference between the corrugations on both surfaces were set to $a=250 \mathrm{~nm}$ and $\varphi=\pi / 2$, respectively. The system was enclosed in a grounded rectangular box which represents the boundary conditions at infinity. Next, boundary conditions (applied voltages) identical to the experimental parameters were assigned to all objects. The rectangular enclosure and the sphere were both set to be grounded. The rectangular box was automatically set to be at infinity by Comsol Multiphysics. Next the Poisson equation was solved for the given boundary conditions. Then the normal electrostatic force was calculated by integrating the $z z$-component of the Maxwell stress tensor over the surface of corrugated sphere and compared with Eq. (17). To make sure that the solution converged, the size of the corrugated objects and the number of the surface mesh elements were both varied and the force recalculated. For each $l$, the number of the mesh elements was increased from one million to 22 millions until the calculated force converged. The size of the truncated section of the corrugated plate and sphere was increased from $l=14 \mu \mathrm{m}$ to $l=50 \mu \mathrm{m}$. The convergence of the force was observed for $l>45 \mu \mathrm{m}$. The corresponding difference of Eq. (7) from the FEA results varied from $-36 \%$ to $2.8 \%$ when $l$ increased from $14 \mu \mathrm{m}$ to $50 \mu \mathrm{m}$, respectively, with convergence at the largest values of $l$. Thus, we confirmed from simulations that Eq. (77) is valid with an error less than $2.8 \%$. It turns out that this error is almost phase independent and, thus, does not propagate to the lateral electric force.

Now we return to the experimental electrostatic calibrations using the normal electric force. The deflection signal $S_{\text {nor }}^{\mathrm{el}}$ was measured for eight different voltages between $-0.52 \mathrm{~V}$ and $0.47 \mathrm{~V}$ applied to the grating at the constant grating-sphere separation $a=1 \mu \mathrm{m}$ where the Casimir force is negligibly small (see below for the determination of absolute separations using the lateral electrostatic force). The respective experimental normal electrostatic force 
is given by

$$
F_{\text {nor }}^{\mathrm{el}}(a, \varphi)=k_{\mathrm{tor}} S_{\text {nor }}^{\mathrm{el}}(a, \varphi)
$$

where $k_{\text {tor }}$ is the normal force calibration constant measured in the units of force per unit deflection signal (note that for the attractive force the deflection signal is negative). This constant is connected with the torsional spring constant discussed above as

$$
k_{\mathrm{tor}}=K_{\mathrm{tor}} m_{\mathrm{tor}}
$$

where the deflection coefficient $m_{\text {tor }}$ is measured in the units of length per unit deflection signal. The obtained experimental data for $S_{\text {nor }}^{\mathrm{el}}$ and for the respective force $F_{\text {nor }}^{\mathrm{el}}$ from Eq. (8) was fitted to Eq. (7). The resulting mean values of $V_{0}$ and $k_{\text {tor }}$ found from the fit are

$$
V_{0}=-39.6 \pm 1.6 \mathrm{mV}, \quad k_{\text {tor }}=7.00 \pm 0.08 \mathrm{nN} / \text { unit } S \text {. }
$$

Measurement of the lateral Casimir force as a function of absolute separation requires knowledge of the lateral force calibration constant

$$
k_{\text {ben }}=K_{\text {ben }} m_{\text {ben }},
$$

where $m_{\text {ben }}$ is the bending deflection coefficient. If the separation on contact $z_{0}$ is determined, absolute separation between the mean values of the corrugations on both surfaces is given by [4, 11]

$$
a=z_{0}+z_{\text {piezo }}+S_{\text {nor }} m_{\text {tor }}
$$

where $z_{\text {piezo }}$ is the distance moved by the plate owing to the voltage applied to the piezoelectric actuator, $S_{\text {nor }}$ is the photodiode difference signal due to the force (either electric or Casimir).

The determination of $k_{\text {ben }}$ and $z_{0}$ is achieved by measuring the cantilever deflection signal due to the lateral electrostatic force which arises when a voltage is applied to the grating. The measurements of this signal were performed at small separations from close to $z_{0}$ to $z_{0}+120 \mathrm{~nm}$. Note that calibrations using the lateral electrostatic force were done after the measurements of the deflection signal due to the lateral Casimir force are performed, but are reported in this section for the benefit of the reader. First, a voltage of $141.456 \mathrm{mV}$ was applied to the grating. The sphere was kept at a distance $3.96 \mathrm{~nm}$ from $z_{0}$. The phase shift between corrugations was changed continuously at a frequency $0.103 \mathrm{~Hz}$ with the $x$-piezo to a maximum translation of $3.3 \mu \mathrm{m}$. 
It is important that corrugated surfaces move parallel to each other as the phase is changed with the $x$-piezo. The grating template was mounted as parallel as possible to the $x$-axis on the AFM piezo. However, experimentally there is always a finite nonzero angle between the grating template and the $x$-axis. When the phase change is introduced by moving the $x$-piezo, this tilt will lead to changes in the separation distance between the corrugated surfaces, which in turn would result in a systematic error of decreasing or increasing force with phase. This nonparallelity should be corrected before start of the experiments using the lateral electrostatic force. A voltage is applied to the plate and the lateral electrostatic force is monitored as a function of the phase over many periods of the grating. A correcting voltage adapted from that applied to the $x$-piezo using an adjustable voltage divider was synchronously applied to the $z$-piezo. The applied voltage to the $z$-piezo was changed till the amplitude of the lateral electrostatic force become independent of the phase. As an example, in Fig. 6 we show the deflection signal due to the lateral electrostatic force versus the phase difference $x_{0}$ between the corrugated surfaces (a) before and (b) after the tilt correction was introduced. This was done approximately $25 \mathrm{~nm}$ away from contact with a $0.2 \mathrm{~V}$ applied voltage. It can be seen that the amplitudes of each peak are more identical in Fig. 6(b). This procedure experimentally verifies that the separation distance between the corrugations remains fixed as the phase was changed with the $x$-piezo.

The cantilever deflection signal $S_{\text {lat }}^{\text {tot }}$ corresponding to the total force acting at the separation $a=z_{0}+3.96 \mathrm{~nm}$ (sum of the lateral electrostatic and lateral Casimir forces) was recorded at 8192 evenly spaced data points. The sphere was moved further away from the grating by $5.40 \mathrm{~nm}$ to a separation $9.36 \mathrm{~nm}$ from $z_{0}$, and the measurement was repeated. The cantilever deflection signal $S_{\text {lat }}^{\text {tot }}$ was measured at many other $a$ at the same voltage and also for a second voltage of $101.202 \mathrm{mV}$ applied to the grating. To obtain the deflection signal due to the lateral electrostatic force alone, it is necessary to subtract from the obtained signal $S_{\text {lat }}^{\text {tot }}$ the deflection signal $S_{\text {lat }}^{\mathrm{C}}$ due to the lateral Casimir force (the signal $S_{\text {lat }}^{\mathrm{C}}$ was measured before $S_{\text {lat }}^{\text {tot }}$ and the respective measurement data are reported in Sec. IV). To perform this subtraction, $S_{\text {lat }}^{\mathrm{C}}$ was fitted to the following sum of harmonics:

$$
S_{\text {lat }}^{\mathrm{C}}(a, \varphi)=\sum_{k=1}^{5} A_{k}(a) \sin (k \varphi),
$$

which takes proper account of the fact that the lateral Casimir force is asymmetric (see Sec. IV). As the sphere-grating separations in the measurements of the lateral Casimir force 
and of the total force are not identical, interpolation was used to determine the values of $A_{k}(a)$ at the separations corresponding to the total lateral force. After determination of the coefficients $A_{k}(a)$ from the fit, the signal $S_{\text {lat }}^{\mathrm{C}}$ was subtracted from the data for the total

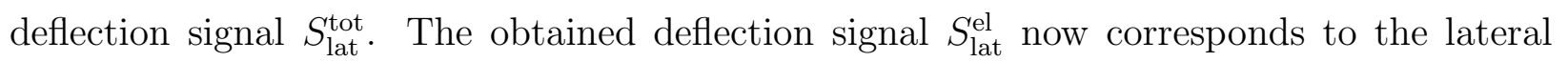
electrostatic force

$$
F_{\text {lat }}^{\mathrm{el}}(a, \varphi)=k_{\mathrm{ben}} S_{\text {lat }}^{\mathrm{el}}(a, \varphi)
$$

The experimental force data in this equation were used to determine the calibration parameter $k_{\text {ben }}$ and $z_{0}$ from the fit to the theoretical expression for the lateral electric force.

To derive the analytic expression for the lateral electric force acting between the sinusoidally corrugated surfaces of a sphere and a plate, we start with the electrostatic energy in sphere-plane configuration with smooth surfaces [47, 48]

$$
E^{\mathrm{el}, \mathrm{sp}}(a)=-2 \pi \epsilon_{0}\left(V-V_{0}\right)^{2} R\left[c_{-1} \ln \frac{R}{a}+\tilde{c}-\sum_{i=0}^{6} \frac{c_{i}}{i+1}\left(\frac{a}{R}\right)^{i+1}\right] .
$$

Here, the integration constant $\tilde{c}$ is equal to [47]

$$
\tilde{c}=\frac{1}{2} \ln 2+\frac{23}{40}+\frac{\theta}{126}
$$

where $0 \leq \theta \leq 1$, and $c_{i}$ are defined in Eq. (21).

Using the PFA, we obtain the electrostatic energy between sinusoidally corrugated surfaces of a sphere and a plate in the form

$$
E^{\mathrm{el}}(a, \varphi)=\frac{1}{\Lambda} \int_{0}^{\Lambda} d x E^{\mathrm{el}, \mathrm{sp}}\left(z_{s}-z_{p}\right)
$$

where $z_{s}-z_{p}$ is given by Eqs. (3), (5) and (6). Using the definition of the lateral electric force [see the formula for the phase shift below Eq. (33)]

$$
F_{\text {lat }}^{\mathrm{el}}(a, \varphi)=-\frac{\partial}{\partial x_{0}} E^{\mathrm{el}}(a, \varphi)=-\frac{2 \pi}{\Lambda} \frac{\partial}{\partial \varphi} E^{\mathrm{el}}(a, \varphi)
$$

and calculating all integrals in (17), we arrive at the result

$$
\begin{aligned}
F_{\text {lat }}^{\mathrm{el}}(a, \varphi) & =\pi^{2} \epsilon_{0}\left(V-V_{0}\right)^{2} R \frac{A_{1} A_{2}}{a^{2} \Lambda}\left[\frac{2}{\sqrt{1-\beta^{2}}\left(1+\sqrt{1-\beta^{2}}\right)}\right. \\
& \left.-2 \sum_{i=1}^{6} i c_{i}\left(\frac{a}{R}\right)^{i+1} Y_{i}\right] .
\end{aligned}
$$


Here, the parameter $\beta \equiv \beta(a, \varphi)$ is defined in Eq. (6) and the coefficients $Y_{i} \equiv Y_{i}\left(\beta^{2}\right)$ are given by

$$
\begin{aligned}
& Y_{1}=Y_{2}=1, \quad Y_{3}=1+\frac{1}{4} \beta^{2}, \quad Y_{4}=1+\frac{3}{4} \beta^{2}, \\
& Y_{5}=1+\frac{4}{3} \beta^{2}+\frac{1}{8} \beta^{4}, \quad Y_{6}=1+10 \beta^{2}+\frac{5}{8} \beta^{4} .
\end{aligned}
$$

By fitting the force data in Eq. (14) to Eq. (19), the quantities $k_{\text {ben }}$ and $z_{0}$ were found. This was repeated for four different electrostatic force measurements and the average values obtained are

$$
z_{0}=117.3 \pm 3.0 \mathrm{~nm}, \quad k_{\text {ben }}=1.27 \pm 0.06 \mathrm{nN} / \text { unit } S .
$$

Using an independent quad-cell AFM measurement of the torsional movement of such a cantilever, the torsional spring constant was found to be $K_{\text {tor }}=0.28 \pm 0.5 \mathrm{~N} / \mathrm{m}$. The value of the bending deflection coefficient $m_{\text {ben }}=209 \pm 3 \mathrm{~nm} /$ unit $S$ was obtained in the same way as in normal Casimir force measurements [42, 49] from the change in position of the contact point for application of different voltages to the plate during the normal electrostatic force measurement. Then the value of the bending spring constant $K_{\text {ben }}=(6.1 \pm 0.3) \times 10^{-3} \mathrm{~N} / \mathrm{m}$ was found from Eqs. (11) and (21). By comparing the two spring constants, we find that $K_{\text {tor }} / K_{\text {ben }} \approx 46$ which means that our system is really much more sensitive to the lateral rather than to the normal Casimir force.

In recent literature [50] there is a discussion that in several measurements of the Casimir force the residual potential difference $V_{0}$ depends on separation, whereas in some other it is constant. Because of this, we have performed an additional control measurement of $V_{0}$ at short separation $a=127.3 \mathrm{~nm}$ using the parabolic dependence of $F_{\text {lat }}^{\mathrm{el}}$ in Eq. (19) on $V$. For this purpose five different voltages close to the residual potential difference were applied to the grating and the cantilever deflection was measured as a function of $\mathrm{V}$ leading to $V_{0}=-39.4 \mathrm{mV}$. This is consistent with the result (10) obtained at large separation $a=1 \mu \mathrm{m}$ and confirms that in our experiment the residual potential difference is separation independent.

The same calibration procedures, as described above for the first set of measurements, were repeated for a second set with a larger corrugation amplitude of $25.5 \mathrm{~nm}$ on the sphere. Here we obtained a residual potential difference $\tilde{V}_{0}=-28.35 \pm 1.15 \mathrm{mV}$ and the following 
values of the separation on contact and of the lateral force calibration constant

$$
\tilde{z}_{0}=131.4 \pm 3.8 \mathrm{~nm}, \quad \tilde{k}_{\text {ben }}=2.05 \pm 0.11 \mathrm{nN} / \text { unit } S
$$

Typically in our second set of measurements with the larger amplitude corrugations the calibration errors are a bit larger than in the first, where smaller amplitude corrugations were used.

\section{MEASUREMENT DATA FOR THE LATERAL CASIMIR FORCE AND ER- ROR ANALYSIS}

Now we describe how the measurements of the lateral Casimir force were performed. In this case the residual voltage $V_{0}$, as determined in the electrostatic calibration using the normal electric force, was applied to the grating in order to make the electric force equal to zero. The $x$-piezo was used to move the grating along the $x$-axis and thus change $\varphi$. The z-piezo, which was independently controlled by an external voltage source, was used to change $a$. The piezo extensions with applied voltage in both directions were calibrated using optical interferometry [51].

Initially the corrugated sphere was positioned $3.79 \mathrm{~nm}$ from the separation on contact between the two surfaces $z_{0}$ determined by the corrugations and the highest roughness peaks. The thermomechanical drift of the separation distance was measured to be $0.14 \mathrm{~nm} / \mathrm{min}$ from the difference in the $z$-piezo voltage to bring about contact of the two corrugated surfaces after a time interval around 30 minutes. A phase shift was introduced by moving the $x$-piezo continuously for a total distance of $3.3 \mu \mathrm{m}$ at $0.103 \mathrm{~Hz}$. The photodiode signal corresponding to the cantilever deflection was filtered with a low-pass filter with a $30 \mathrm{~ms}$ time constant and recorded at each of the 8192 points corresponding to $x$-changes of $0.4 \mathrm{~nm}$. The effect of the scattered laser light which would lead to a linear modification of the signal with phase was found to be negligible in this experiment.

Then the separation from $z_{0}$ was increased by $3.6 \mathrm{~nm}$ from $3.79 \mathrm{~nm}$ to $7.39 \mathrm{~nm}$ and the deflection signal $S_{\text {lat }}^{\mathrm{C}}$ was similarly measured as a function of $\varphi$ and recorded. After this, the separation from $z_{0}$ was increased by $3.96 \mathrm{~nm}$ from $7.39 \mathrm{~nm}$ to $11.35 \mathrm{~nm}$ and the measurements

repeated. Next $S_{\text {lat }}^{\mathrm{C}}$ due to the lateral Casimir force as a function of $\varphi$ was measured at separations of $20.05,32.48,45.30,58.01$ and $70.86 \mathrm{~nm}$ from $z_{0}$. 
Using the values of $k_{\text {ben }}$ and $z_{0}$ obtained through the electrostatic calibration (see Sec. III) the measurement data for the deflection signal $S_{\text {lat }}^{\mathrm{C}}$ were converted into values of the lateral Casimir force at every separation $a$ as:

$$
F_{\text {lat }}^{\mathrm{C}}(a, \varphi)=k_{\text {ben }} S_{\text {lat }}^{\mathrm{C}}(a, \varphi)
$$

The resulting lateral Casimir force for a sphere with the corrugation amplitude $A_{2}=13.7 \mathrm{~nm}$ over four corrugation periods is shown in Fig. 7(a-c) as dots versus the normalized lateral displacement $x / \Lambda$ between the corrugated surfaces at separations $a=124.7,128.6$, and $149.8 \mathrm{~nm}$, respectively. Similar results were obtained at all other $a$ listed above. As can be seen in Fig. 7, the lateral Casimir force is a periodic function of the phase shift. The maximum values of the lateral force, $f \equiv \max \left|F_{\text {lat }}^{\mathrm{C}}\right|$, decrease with the increase of $a$. The important characteristic feature of the periodic curves in Fig. 7(a-c) is that they are asymmetric, i.e., the dependence of $F_{\text {lat }}^{\mathrm{C}}$ on $x / \Lambda$ is not strictly sinusoidal (note that the sinusoidal dependence of the lateral force on the phase shift holds only if the calculation is restricted to the lowest order in corrugation amplitudes $\sim A_{1} A_{2}$; see Sec. V), The true asymmetry of the measured lateral force is obvious even without the theory curve. For example, in Fig. 7(a) the average shift of each maximum point from the midpoint of two adjacent minima is $(0.12 \pm 0.2) \Lambda$. With the increase of separation, the relative contribution of higher perturbation orders in a small parameter $A_{1} A_{2} / a^{2}$ decreases and the dependence of the lateral force on the phase shift becomes more close to sinusoidal. The solid lines in Fig. 7 are related to the exact theoretical computations. They are discussed in Sec. VI.

The experimental values of the $\max \left|F_{\text {lat }}^{\mathrm{C}}\right|$ versus separation $a$ are shown in Fig. 8 as crosses. The arms of the crosses indicate the total experimental errors determined at a 95\% confidence level using the following procedure. From the data of the phase curves in Fig. 7 (and related curves at all the other separations measured) we have calculated the mean values $\bar{f}$ and the variances $s_{\bar{f}}$ of the quantity $f=\max \left|F_{\text {lat }}^{\mathrm{C}}\right|$ at each $a$ (see columns 2 and 3 in Table 1). For the first set of measurements under consideration, the averaging was performed over the four periods. The respective Student coefficient is $t_{(1+\gamma) / 2}(3)=3.18$ where $\gamma=0.95$. Thus, the experimental random error is obtained from the data in column 3 of Table 1 as

$$
\Delta^{\mathrm{rand}} f(a)=s_{\bar{f}} t_{0.975}(3)
$$

The main sources of systematic errors in the quantity $f$ are the errors due to the uncertainty 
in $k_{\text {ben }}$ and due to the resolution of data. According to Eq. (21),$\Delta k_{\text {ben }} / k_{\text {ben }}=0.047$. From Eq. (23) this leads to the systematic error $\Delta_{1}^{\text {syst }} f(a)=0.047 f(a)$. The systematic error resulting from the resolution of data does not depend on separation $\Delta_{2}^{\text {syst }} f(a)=0.035 \mathrm{pN}$. Combining these two errors at a 95\% confidence level as random quantities characterized by a uniform distribution [2, 52, 53], we obtain the values of the total systematic error given in column 4 of Table 1 . Using the rule for the combination of random and systematic errors at the same confidence level [2, 52, 53], we obtain the total experimental error $\Delta^{\text {tot }} f$ as a function of separation presented in the fifth column of Table 1. As can be seen in Table 1, all absolute errors are larger at shorter separations and decrease with increasing separation. This might be explained by local deviations of the shape of corrugations from perfect sinusoidal form which are more influential at shorter separations. The total relative error of $\max \left|F_{\text {lat }}^{\mathrm{C}}\right|$ at $a=121.1 \mathrm{~nm}$ is equal to $22.5 \%$ (at a $95 \%$ confidence level). The relative errors at separations $a=124.7,128.6$, and $137.3 \mathrm{~nm}$ are equal to $13 \%, 25.5 \%$, and $14 \%$, respectively.

The error in the measurement of the absolute separations, $\Delta a$, is a combination of the error in the determination of $z_{0}, \Delta z_{0}=3 \mathrm{~nm}$, indicated in Eq. (21) and the error in the quantity $z_{\text {piezo }}+S_{\text {nor }} m_{\text {tor }}$ in Eq. (12). The latter is equal to the half of the first step of the $z$-piezo, $\Delta_{p} z \approx 2 \mathrm{~nm}$. Combining these two errors at a $95 \%$ confidence level with the help of the same rule, as applied above for the systematic errors [2, 52, 53], one obtains $\Delta a=4 \mathrm{~nm}$. This is a factor of eight improvement as compared with Ref. [38]. Note that the bands between the dashed and the solid lines in Fig. 8 are related to theoretical predictions using the PFA and the exact theory, respectively. They are discussed in Secs. V and VI.

The same measurement procedure, as described above, was applied in the second set of measurements for a sphere covered with deeper corrugations of an amplitude $\tilde{A}_{2}$. The resulting lateral Casimir force over five or six corrugation periods is shown in Fig. 9(a-c) as dots versus the lateral displacement at separations $a=134,156.5$ and $179 \mathrm{~nm}$, respectively. Similar results were obtained also at separations $a=145.2,201.6,224$, and $246.6 \mathrm{~nm}$. In the same way, as in Fig. 7, the lateral Casimir force at each $a$ is a periodic function which is asymmetric (nonsinusoidal). The asymmetry is more pronounced at shorter separations. The experimental values of the $\max \left|F_{\text {lat }}^{\mathrm{C}}\right|$ versus separation are plotted in Fig. 10 as crosses. Similar to Fig. 8 the arms of the crosses show the total experimental errors of force and separation measurements determined at a 95\% confidence level. Equation (22) and the 
same statistical procedure, as in the first set of measurements, were used to determine these errors with the only difference that for the second set the averaging was performed at all separations over five periods [the respective Student coefficient is $t_{(1+\gamma) / 2}(4)=2.78$ with $\gamma=0.95]$. The total error in the measurement of separation, $\Delta a=4.7 \mathrm{~nm}$, turned out to be a bit larger than in the first set of measurements. The total error of the lateral force measurements demonstrates similar irregular behavior on separation distance. This can also be explained by the influence of local deviations of groove shape from sinusoidal form keeping in mind that with larger corrugation amplitude the role of such deviations should be larger. In Table 2 we present the mean values, the variances of the mean, the systematic errors, and the total experimental errors (at a 95\% confidence level) of the maximum magnitudes of the lateral Casimir force at different separations for the second set of measurements. The total relative error of the lateral force measurements at separations $a=134,145.2,156.5$, and $179 \mathrm{~nm}$ varies as $23 \%, 16 \%, 14 \%$, and $13 \%$, respectively, all calculated at a $95 \%$ confidence level.

\section{COMPUTATION OF THE LATERAL CASIMIR FORCE USING THE PROX- IMITY FORCE APPROXIMATION}

In this section and in Sec. VI we compare the obtained experimental results for the lateral Casimir force with two theoretical approaches applicable in the case of corrugated surfaces: the PFA and the exact scattering approach, respectively, with no fitting parameters. As was mentioned in the Introduction, the PFA approach was also used to compare with theory the measurement data of the first observation of the lateral Casimir force in Refs. [37, 38]. However, in those papers the real properties of $\mathrm{Au}$ were described using the simple plasma model and fourth-order perturbation theory with respect to the relative skin depth at zero temperature. Here, we develop a more complete description of the experimental data in the framework of the PFA approach based on the Lifshitz formula at the laboratory temperature. This will help us to separate diffraction type contributions to the lateral Casimir force which are beyond the PFA.

We start with the Lifshitz formula for the normal Casimir force acting between a sphere 
and a plate made of a real material $(\mathrm{Au})$ but bounded with perfectly smooth surfaces [2, 8, 11]

$$
\begin{aligned}
F^{\mathrm{C}, \mathrm{sp}}(a) & =k_{B} T R \sum_{l=0}^{\infty} \int_{0}^{\infty} k_{\perp} d k_{\perp}\left\{\ln \left[1-r_{\mathrm{TM}}^{2}\left(i \xi_{l}, k_{\perp}\right) e^{-2 q_{l} a}\right]\right. \\
& \left.+\ln \left[1-r_{\mathrm{TE}}^{2}\left(i \xi_{l}, k_{\perp}\right) e^{-2 q_{l} a}\right]\right\} .
\end{aligned}
$$

Here, $k_{B}$ is the Boltzmann constant, $\xi_{l}=2 \pi k_{B} T l / \hbar$ with $l=0,1,2, \ldots$ are the Matsubara frequencies, prime near the summation sign adds a multiple $1 / 2$ to the term with $l=0$. The reflection coefficients for the transverse magnetic and transverse electric polarizations of the electromagnetic field are defined as

$$
r_{\mathrm{TM}}\left(i \xi_{l}, k_{\perp}\right)=\frac{\varepsilon_{l} q_{l}-k_{l}}{\varepsilon_{l} q_{l}+k_{l}}, \quad r_{\mathrm{TE}}\left(i \xi_{l}, k_{\perp}\right)=\frac{q_{l}-k_{l}}{q_{l}+k_{l}}
$$

where

$$
q_{l}^{2}=k_{\perp}^{2}+\frac{\xi_{l}^{2}}{c^{2}}, \quad k_{l}^{2}=k_{\perp}^{2}+\varepsilon_{l} \frac{\xi_{l}^{2}}{c^{2}}
$$

and $\varepsilon_{l} \equiv \varepsilon\left(i \xi_{l}\right)$ is the dielectric permittivity for the material of the sphere and plate calculated along the imaginary Matsubara frequencies. Equation (25) is obtained from the standard Lifshitz expression for the free energy in the configuration of two parallel plates by the multiplication by $2 \pi R$ in accordance with the PFA. Keeping in mind the values of the experimental parameters $(R \sim 100 \mu \mathrm{m}, a \sim 100 \mathrm{~nm})$, one can conclude that the error introduced by the use of the PFA in this case (of about $a / R=0.1 \%[8,[54[56]$ ) is negligibly small.

Now we consider the sphere and the plate covered with sinusoidal corrugations described in Secs. II and III. Within the PFA approach, the approximate expression for the normal Casimir force acting between corrugated surfaces of a sphere and a plate can be obtained from Eq. (25) by replacing $a$ with $z_{s}-z_{p}$ defined in Eq. (3) and averaging over the period of corrugations $\Lambda$. By expanding also the logarithms in Eq. (25) into a series, we arrive at

$$
\begin{aligned}
& F_{\text {nor }}^{\mathrm{C}}(a, \varphi)=-\frac{k_{B} T R}{\Lambda} \sum_{n=1}^{\infty} \frac{1}{n} \sum_{l=0}^{\infty} \int_{0}^{\infty} k_{\perp} d k_{\perp} \\
& \quad \times\left[r_{\mathrm{TM}}^{2 n}\left(i \xi_{l}, k_{\perp}\right)+r_{\mathrm{TE}}^{2 n}\left(i \xi_{l}, k_{\perp}\right)\right] e^{-2 q_{l} n a} \\
& \quad \times \int_{0}^{\Lambda} d x e^{-2 q_{l} n\left[A_{2} \sin (2 \pi x / \Lambda+\varphi)-A_{1} \sin (2 \pi x / \Lambda)\right]} .
\end{aligned}
$$

From this expression it is simple to obtain the Casimir energy in the configuration of a 
sphere above a plate both covered with sinusoidal corrugations

$$
\begin{aligned}
& E^{\mathrm{C}}(a, \varphi)=\int_{a}^{\infty} d z F_{\mathrm{nor}}^{\mathrm{C}}(z, \varphi) \\
& =-\frac{k_{B} T R}{2 \Lambda} \sum_{n=1}^{\infty} \frac{1}{n^{2}} \sum_{l=0}^{\infty} \int_{0}^{\infty} \frac{k_{\perp} d k_{\perp}}{q_{l}} \\
& \quad \times\left[r_{\mathrm{TM}}^{2 n}\left(i \xi_{l}, k_{\perp}\right)+r_{\mathrm{TE}}^{2 n}\left(i \xi_{l}, k_{\perp}\right)\right] e^{-2 q_{l} n a} \\
& \quad \times \int_{0}^{\Lambda} d x e^{-2 q_{l} n\left[A_{2} \sin (2 \pi x / \Lambda+\varphi)-A_{1} \sin (2 \pi x / \Lambda)\right]} .
\end{aligned}
$$

Introducing the dimensionless variables

$$
y=2 a q_{l}, \quad \zeta_{l}=\frac{2 a \xi_{l}}{c}
$$

and performing the integration with respect to $x$, one can rearrange Eqs. (28) and (29) to the form

$$
\begin{aligned}
& F_{\text {nor }}^{\mathrm{C}}(a, \varphi)=-\frac{k_{B} T R}{4 a^{2}} \sum_{n=1}^{\infty} \frac{1}{n} \sum_{l=0}^{\infty}{ }^{\prime} \int_{\zeta_{l}}^{\infty} y d y \\
& \quad \times\left[r_{\mathrm{TM}}^{2 n}\left(i \zeta_{l}, y\right)+r_{\mathrm{TE}}^{2 n}\left(i \zeta_{l}, y\right)\right] e^{-n y} I_{0}(n \beta y), \\
& E^{\mathrm{C}}(a, \varphi)=-\frac{k_{B} T R}{4 a} \sum_{n=1}^{\infty} \frac{1}{n^{2}} \sum_{l=0}^{\infty} \int_{\zeta_{l}}^{\infty} d y \\
& \quad \times\left[r_{\mathrm{TM}}^{2 n}\left(i \zeta_{l}, y\right)+r_{\mathrm{TE}}^{2 n}\left(i \zeta_{l}, y\right)\right] e^{-n y} I_{0}(n \beta y) .
\end{aligned}
$$

Here, $I_{0}(z)$ is the Bessel function of an imaginary argument and $\beta$ is defined in Eq. (6).

The general expression for the lateral Casimir force can be obtained from Eq. (32) in the same way as the lateral electric force in Eq. (18). After performing the differentiation, we rearrange the result to the form

$$
\begin{aligned}
& F_{\text {lat }}^{\mathrm{C}}(a, \varphi)=\frac{\pi k_{B} T R A_{1} A_{2}}{2 a^{3} \Lambda \beta} \sin \varphi \sum_{n=1}^{\infty} \frac{1}{n} \sum_{l=0}^{\infty} \int_{\zeta_{l}}^{\infty} y d y \\
& \times\left[r_{\mathrm{TM}}^{2 n}\left(i \zeta_{l}, y\right)+r_{\mathrm{TE}}^{2 n}\left(i \zeta_{l}, y\right)\right] e^{-n y} I_{1}(n \beta y) .
\end{aligned}
$$

This expression is convenient for numerical computations.

Equations (31) and (33) are generalizations for the case of real materials of the previously known expressions obtained at $T=0$ for ideal metal corrugated bodies. In this case $r_{\mathrm{TM}}^{2}=$ $r_{\mathrm{TE}}^{2}=1$ and one obtains

$$
\begin{aligned}
& F_{\text {nor }}^{\mathrm{C}}(a, \varphi)=-\frac{\hbar c R}{8 \pi a^{3}} \sum_{n=1}^{\infty} \frac{1}{n} \int_{0}^{\infty} y^{2} d y e^{-n y} I_{0}(n \beta y), \\
& F_{\text {lat }}^{\mathrm{C}}(a, \varphi)=\frac{\hbar c R A_{1} A_{2}}{4 a^{4} \Lambda \beta} \sin \varphi \sum_{n=1}^{\infty} \frac{1}{n} \int_{0}^{\infty} y^{2} d y e^{-n y} I_{1}(n \beta y) .
\end{aligned}
$$


Introducing the new variable $v=n y$ and performing the summation, we arrive at

$$
\begin{aligned}
& F_{\text {nor }}^{\mathrm{C}}(a, \varphi)=-\frac{\pi^{3} \hbar c R}{720 a^{3}} \int_{0}^{\infty} d v v^{2} e^{-v} I_{0}(\beta v), \\
& F_{\text {lat }}^{\mathrm{C}}(a, \varphi)=\frac{\pi^{4} \hbar c R A_{1} A_{2}}{360 a^{4} \Lambda \beta} \sin \varphi \int_{0}^{\infty} d v v^{2} e^{-v} I_{1}(\beta v) .
\end{aligned}
$$

After performing the integration in these equations, the final result is

$$
\begin{aligned}
& F_{\text {nor }}^{\mathrm{C}}(a, \varphi)=-\frac{\pi^{3} \hbar c R}{720 a^{3}} \frac{2+\beta^{2}}{\left(1-\beta^{2}\right)^{5 / 2}}, \\
& F_{\text {lat }}^{\mathrm{C}}(a, \varphi)=\frac{\pi^{4} \hbar c R A_{1} A_{2}}{360 a^{4} \Lambda\left(1-\beta^{2}\right)^{5 / 2}} \sin \varphi
\end{aligned}
$$

The last expression for the lateral force was obtained in Refs. [37, 38] with corrections due to the skin depth. In the first perturbation order in $A_{1} A_{2}$ (i.e., for $\beta=0$ ) this expression was also used in Refs. [24, 25].

Equations (31) and (33) take into account the sphericity of the second test body and sinusoidal corrugations on both bodies in the framework of the PFA and the nonzero skin depth in the framework of the Lifshitz theory. To perform numerical computations, one should use a model to represent the dielectric permittivity. At the separations considered (from 100 to $250 \mathrm{~nm}$ ) the thermal effects do not play any role in any of the theoretical approaches and reliable results can be obtained using the generalized plasma-like model $[2,39,40]$

$$
\varepsilon\left(i \xi_{l}\right)=1+\frac{\omega_{p}^{2}}{\xi_{l}^{2}}+\sum_{j=1}^{6} \frac{g_{j}}{\omega_{j}^{2}+\xi_{l}^{2}+\gamma_{j} \xi_{l}},
$$

where $\omega_{p}=9.0 \mathrm{eV}$ for $\mathrm{Au}$ is the plasma frequency. The values of the oscillator frequencies $\omega_{j}$, relaxation parameters $\gamma_{j}$ and the oscillator strengths $g_{j}$ leading to approximately the same results for $\operatorname{Im} \varepsilon(\omega)$ as those obtained from the optical tabulated data [57] over the frequency range from 2 to $25 \mathrm{eV}$ are listed in Refs. [2, 40].

An important factor that is not taken into account in Eq. (33) is the surface roughness. Stochastic roughness covering the corrugated surfaces in both the sphere and the plate is clearly seen in Figs. 2, 3 and its measured variances are presented in Sec. II. In view of its stochastic (nonperiodic) character, surface roughness cannot influence the phase dependence of the Casimir energy in the configuration of corrugated surfaces. However, it contributes to the Casimir energy, and, thus, to the lateral Casimir force, through a phase independent correction factor which depends only on separation. It has been found [2, 8] that the 
correction factor to the Casimir force due to stochastic roughness in the configuration of a sphere above a plate with no corrugations is given by

$$
\eta_{F}(a)=1+6 \frac{\delta_{1}^{2}+\delta_{2}^{2}}{a^{2}}+45 \frac{\left(\delta_{1}^{2}+\delta_{2}^{2}\right)^{2}}{a^{4}} .
$$

Now we take into account that in the framework of the multiplicative approach the correction factor due to surface roughness is the same for real and ideal metals. Keeping in mind that the Casimir force between a perfectly shaped ideal metal sphere and a plate is $F(a) \sim 1 / a^{3}$, we obtain the correction to the Casimir energy by integrating the Casimir force with the inclusion of roughness, $\eta_{F}(a) F(a)$, with respect to $a$

$$
\eta_{E}(a)=1+3 \frac{\delta_{1}^{2}+\delta_{2}^{2}}{a^{2}}+15 \frac{\left(\delta_{1}^{2}+\delta_{2}^{2}\right)^{2}}{a^{4}} .
$$

Note that for the experimental variances (see Sec. II) the fourth order term in Eq. (39) is negligibly small. Thus, for the first set of measurements at the shortest separation $a=$ $120 \mathrm{~nm}$ the second order term contributes $3 \%$ of the force, but the fourth order term only $0.2 \%$ of the force. For the second set of measurement with deeper corrugations on the sphere the contributions of the second and fourth order terms at separation $a=134 \mathrm{~nm}$ are $4 \%$ and $0.3 \%$, respectively.

To obtain the correction factor to the lateral Casimir force due to the surface roughness with account of the sinusoidal corrugations on the sphere and on the plate, we should replace $a$ with separation $z_{s}-z_{p}$ between the corrugated surfaces in Eqs. (39) and (5) and perform the averaging with respect to the phase shift and the period

$$
\begin{aligned}
& \eta_{\text {corr }}(a)=1+3\left(\delta_{1}^{2}+\delta_{2}^{2}\right)\left\langle\frac{1}{\left(z_{s}-z_{p}\right)^{2}}\right\rangle \\
& \left\langle\frac{1}{\left(z_{s}-z_{p}\right)^{2}}\right\rangle=\frac{1}{2 \pi \Lambda a^{2}} \int_{0}^{2 \pi} d \varphi \int_{0}^{\Lambda} d x \frac{1}{[1+\beta \cos (2 \pi x / \Lambda-\alpha)]^{2}}
\end{aligned}
$$

Calculating both integrals in the second equality in Eq. (40) and substituting the obtained result into the first equality, we arrive at

$$
\begin{gathered}
\eta_{\text {corr }}(a)=1+3 \frac{\delta_{1}^{2}+\delta_{2}^{2}}{a\left(a+A_{1}-A_{2}\right)^{1 / 2}\left(a+A_{2}-A_{1}\right)^{1 / 2}} \\
\times F\left(\frac{1}{2}, \frac{1}{2} ; 1 ; \frac{4 A_{1} A_{2}}{\left(a+A_{1}-A_{2}\right)\left(a+A_{2}-A_{1}\right)}\right),
\end{gathered}
$$

where $F(\alpha, \beta ; \gamma ; z)$ is the hypergeometric function. 
Substituting the values of $A_{i}$ and $\delta_{i}$ from the first set of measurements (see Sec. II) in Eq. (41), we find that at separations $a=120,150$, and $200 \mathrm{~nm}$ the correction factor due to surface roughness takes the values $\eta_{\text {corr }}=1.054,1.029$, and 1.015 , respectively. For the second set of measurements (i.e., using $\tilde{A}_{2}$ and $\tilde{\delta}_{2}$ in Sec. II) the correction factor in Eq. (41) takes the values $\tilde{\eta}_{\text {corr }}=1.060,1.041$, and 1.021 at separations $a=130,150$, and $200 \mathrm{~nm}$, respectively. It is seen that even for the second set of our measurements, where the surface roughness on the sphere is larger than in the first one, the roughness correction is rather moderate and does not exceed $6 \%$ of the lateral Casimir force.

In Fig. 8 (the lower dashed line) we present the computational results for the maximum values of the lateral Casimir force as a function of separation in the framework of the PFA [i.e., using Eqs. (33) and (37)] for the first set of our measurements. This line does not take surface roughness into account. The upper dashed line in Fig. 8 presents the results for $\max \left|F_{\text {lat }}^{\mathrm{C}}\right| \eta_{\text {corr }}$ as a function of separation, i.e., taking into account the correction due to surface roughness. Keeping in mind that the multiplicative approach provides the means to estimate the effect of roughness rather than to calculate it precisely on the basis of the fundamental theory, we consider the band between the dashed lines in Fig. 8 as a theoretical prediction given by the PFA approach. As can be seen in Fig. 8, the experimental data indicated as crosses are inconsistent with the prediction of the PFA approach, thus revealing the role of diffraction-type effects on the corrugations of relatively small period used in this experiment.

In Fig. 10 a similar comparison between the experimental data and theory using the PFA approach is done for the second set of our measurements with corrugations of larger amplitude. The lower dashed line is computed using Eqs. (33) and (37) with the surface roughness disregarded. The upper dashed line is obtained by the multiplication of the computation results by an additional factor $\tilde{\eta}_{\text {corr }}$ computed using Eq. (41). Again the theoretical band between the dashed lines is inconsistent with the experimental data shown as crosses due to the neglect of diffraction-like effects. In the next section we present a more fundamental theoretical approach taking the diffraction effects into account which brings theory into agreement with the experimental data. 


\section{COMPUTATION OF THE LATERAL CASIMIR FORCE USING THE RAYLEIGH THEORY}

The PFA approach used to calculate the lateral Casimir force in the configuration of a sphere above a plate with corrugated boundaries has an uncontrolled error which increases with decreasing period of corrugations. As was mentioned in the Introduction, in such cases a more fundamental theory is desirable which takes exact account of diffraction effects. Here, we present such a theory, based on the scattering approach, for the configuration of two plates covered with periodical corrugations of arbitrary shape but common period. This theory can be applied to our experimental configuration by using the PFA for the transition from the configuration of two plane plates to a smooth sphere above a plate. According to our discussion in Sec. V, the error in computations introduced by the use of the PFA for this restricted purpose alone, is extremely small and quite satisfactory for the needs of our experiment.

The scattering approach started with the work [58] where it was applied to obtain the Lifshitz formula and Refs. [59, 60] on the multi-scattering expansion. On this basis the multipole scattering technique was developed in Refs. [61 63] and was applied to several configurations with curved boundaries for scalar and electromagnetic cases. Alternative techniques within the scattering approach to the Casimir effect were presented in Refs. [6467]. For objects of spherical and cylindrical shapes the multipole scattering technique was used in Refs. [54, 55, 68 70]. This technique works well for large separations between the objects and shows poor convergence when the separations between the objects are small. At small separations the leading asymptotic expansion of the Casimir energy at zero temperature within the scattering approach was developed in Ref. [71]. The technique developed in Refs. [35, 36] uses another basis, the Rayleigh basis, which is a generalization of the plane wave basis. The Rayleigh basis is a natural choice for grating geometries.

We consider two parallel 3-dimensional longitudinal (along the $y$-axis) periodic (along the $x$-axis) dielectric (metallic) gratings of arbitrary form separated by a vacuum gap so that they form a waveguide and one grating is located above the other (we assume that the edge of the corrugation region of each grating is perpendicular to the $z$ axis). The periods of both gratings $\Lambda$ are equal. In general, the top and bottom gratings can have different shapes and different dielectric (metallic) properties. In Fig. 11 the height of the corrugation 
region is equal to $h$ for the bottom grating. The meaning of the lateral displacement $x_{0}$ becomes obvious from the comparison of Fig. 11 with Fig. 12 and Fig. 13. We suppose that the space between the two gratings is vacuum with $\varepsilon=\mu=1$ and we assume $\mu=1$ inside the medium.

The physical problem is time and $y$ invariant, so that the particular solutions for the electric and magnetic fields can be written in the form:

$$
\begin{aligned}
& E_{i}(x, y, z, t)=E_{i}(x, z) \exp \left(i k_{y} y-i \omega t\right), \\
& H_{i}(x, y, z, t)=H_{i}(x, z) \exp \left(i k_{y} y-i \omega t\right) .
\end{aligned}
$$

The solutions of Maxwell equations should satisfy the quasi-periodicity conditions:

$$
\begin{aligned}
& E_{i}(x+\Lambda, z)=e^{i k_{x} \Lambda} E_{i}(x, z), \\
& H_{i}(x+\Lambda, z)=e^{i k_{x} \Lambda} H_{i}(x, z) .
\end{aligned}
$$

In every Casimir problem one needs to determine the complete basis of solutions. Let us suppose that the top grating is absent. We consider a generalized conical diffraction problem $\left(k_{y} \neq 0\right)$ on the lower grating for the incident wave with the $x$-component of the wave vector equal to some fixed value which we denote $\tilde{k}_{x}$. The longitudinal components of the electromagnetic field outside the corrugated region $(z \geq h)$ may be written by making use of the Rayleigh expansion [72] for an incident monochromatic wave:

$$
\begin{aligned}
E_{y}(x, z)= & I_{\tilde{k}_{x}}^{(e)} \exp \left(i \tilde{k}_{x} x-i \tilde{\beta}^{(1)} z\right) \\
& +\sum_{n=-\infty}^{\infty} R_{n p}^{(e)} \exp \left(i \alpha_{n} x+i \beta_{n}^{(1)} z\right), \\
H_{y}(x, z)= & I_{\tilde{k}_{x}}^{(h)} \exp \left(i \tilde{k}_{x} x-i \tilde{\beta}^{(1)} z\right) \\
& +\sum_{n=-\infty}^{\infty} R_{n p}^{(h)} \exp \left(i \alpha_{n} x+i \beta_{n}^{(1)} z\right) .
\end{aligned}
$$

Here

$$
\alpha_{n}=k_{x}+2 \pi n / \Lambda
$$

with

$$
k_{x}=\tilde{k}_{x}-\frac{2 \pi}{\Lambda}\left[\frac{\Lambda \tilde{k}_{x}}{2 \pi}\right] \equiv \tilde{k}_{x}-\frac{2 \pi}{\Lambda} p
$$


where $[r]$ is the integer part of the number $r$. From this it follows that $0 \leq k_{x}<2 \pi / \Lambda$. The other notation in Eq. (44) is as follows:

$$
\tilde{\beta}^{(1)^{2}}=\omega^{2}-k_{y}^{2}-\tilde{k}_{x}^{2}, \quad \beta_{n}^{(1)^{2}}=\omega^{2}-k_{y}^{2}-\alpha_{n}^{2},
$$

The quantities $I_{\hat{k}_{x}}^{(e, h)}$ and $R_{n p}^{(e, h)}$ in Eq. (44) are the Rayleigh coefficients. [Note that with notations (45)-(47) it holds $\tilde{k}_{x} \equiv \alpha_{p}, \tilde{\beta}^{(1)} \equiv \beta_{p}^{(1)}$.] The solution (44) is also valid outside any periodic structure in $x$ direction. All other field components can be expressed in terms of the longitudinal components $E_{y}, H_{y}$ by using standard formulas as done in waveguide theory. This can be done since the factor $\exp \left(i k_{y} y\right)$ is conserved after the reflection of the electromagnetic wave from the grating.

At $z=0$ the solution has to satisfy the expansions

$$
\begin{aligned}
& E_{y}(x, z)=\sum_{n=-\infty}^{\infty} T_{n p}^{(e)} \exp \left(i \alpha_{n} x-i \beta_{n}^{(2)} z\right), \\
& H_{y}(x, z)=\sum_{n=-\infty}^{\infty} T_{n p}^{(h)} \exp \left(i \alpha_{n} x-i \beta_{n}^{(2)} z\right),
\end{aligned}
$$

where

$$
\beta_{n}^{(2)}{ }^{2}=\varepsilon \omega^{2}-k_{y}^{2}-\alpha_{n}^{2},
$$

which are valid for $z \leq 0$. The coefficients $T_{n p}^{(e, h)}$ are called the transmission matrix coefficients.

The $E_{y}$ component of the electromagnetic field in the region $0 \leq z \leq h$ is defined as follows:

$$
E_{y}(x, z)=\sum_{n=-\infty}^{\infty} E_{y}^{n}(z) \exp \left(i \alpha_{n} x\right)
$$

The other components of the electromagnetic field in the region $0 \leq z \leq h$ are defined in analogy. Inside the corrugation region $0 \leq z \leq h$ it is convenient to rewrite Maxwell equations in the form of the first order differential equations, $\partial A / \partial y=M(z) A$, where $M(z)$ is a square matrix of dimension $8 N+4, A^{T}=\left(E_{y}^{N} \ldots E_{y}^{-N}, E_{x}^{N} \ldots E_{x}^{-N}, H_{y}^{N} \ldots H_{y}^{-N}, H_{x}^{N} \ldots H_{x}^{-N}\right)$ and $2 N+1$ is the number of coefficients considered in the expansion (50) for $E_{y}$ and similar expansions for $E_{x}, H_{y}$ and $H_{x}$.

For a rectangular grating the matrix $M(z)$ is constant (independent of $z$ ). From Maxwell 
equations

$$
\begin{aligned}
i k_{y} E_{x}-\frac{\partial E_{y}}{\partial x} & =-i \omega H_{z} \\
i k_{y} H_{x}-\frac{\partial H_{y}}{\partial x} & =i \omega \varepsilon E_{z}
\end{aligned}
$$

we get

$$
\begin{aligned}
& E_{z}=\frac{1}{i \omega \varepsilon}\left(i k_{y} H_{x}-\frac{\partial H_{y}}{\partial x}\right), \\
& H_{z}=-\frac{1}{i \omega}\left(i k_{y} E_{x}-\frac{\partial E_{y}}{\partial x}\right) .
\end{aligned}
$$

Substituting into Eq. (52) the expansion (50) and analogous expansions for all other components of $\boldsymbol{E}$ and $\boldsymbol{H}$, we arrive at

$$
\begin{aligned}
E_{z}^{n} & =\sum_{m}\left(\frac{1}{i \omega \varepsilon}\right)_{n-m}\left(i k_{y} H_{x}^{m}-i \alpha_{m} H_{y}^{m}\right), \\
H_{z}^{n} & =-\frac{1}{i \omega}\left(i k_{y} E_{x}^{n}-i \alpha_{n} E_{y}^{n}\right)
\end{aligned}
$$

where $(\Theta)_{n-m}$ is the Toeplitz matrix.

The following equations follow from the remaining four Maxwell equations:

$$
\begin{aligned}
& \frac{d E_{y}^{n}}{d z}=i k_{y} E_{z}^{n}-i \omega H_{x}^{n}, \\
& \frac{d E_{x}^{n}}{d z}=i \alpha_{n} E_{z}^{n}+i \omega H_{y}^{n}, \\
& \frac{d H_{y}^{n}}{d z}=i k_{y} H_{z}^{n}+\sum_{m}(i \omega \varepsilon)_{n-m} E_{x}^{m}, \\
& \frac{d H_{x}^{n}}{d z}=i \alpha_{n} H_{z}^{n}-\sum_{m}(i \omega \varepsilon)_{n-m} E_{y}^{m} .
\end{aligned}
$$

One can substitute Eq. (53) into Eq. (54) and obtain a system of the first order differential equations for the Fourier components of the electromagnetic field $E_{y}^{n}, E_{x}^{n}, H_{y}^{n}, H_{x}^{n}$ in the region $0 \leq z \leq h$.

Now we have to determine the Rayleigh coefficients $R_{n p}^{(e)}, R_{n p}^{(h)}$ for the specific periodic geometry profile. One can determine these coefficients by matching the solution of equations inside the corrugation region $0 \leq z \leq h$ with Rayleigh expansions (44) at $z=h$ and expansions (48) at $z=0$. This can be done by imposing the continuity conditions on each Fourier component of the fields $E_{y}, E_{x}, H_{y}, H_{x}$ at $z=0$ and $z=h$. 
There is no separation of the TE and TM modes for the general case. That is why the reflection matrix $R_{\mathrm{bot}}^{(1)}$ for the reflection from the bottom grating can be defined as follows:

$$
R_{\mathrm{bot}}^{(1)}\left(\boldsymbol{k}_{\perp}, \omega\right)=\left(\begin{array}{ll}
R_{n_{1} q_{1}}^{(e)}\left(I_{p}^{(e)}=\delta_{p q_{1}}, I_{p}^{(h)}=0\right) & R_{n_{2} q_{2}}^{(e)}\left(I_{p}^{(e)}=0, I_{p}^{(h)}=\delta_{p q_{2}}\right) \\
R_{n_{3} q_{3}}^{(h)}\left(I_{p}^{(e)}=\delta_{p q_{3}}, I_{p}^{(h)}=0\right) & R_{n_{4} q_{4}}^{(h)}\left(I_{p}^{(e)}=0, I_{p}^{(h)}=\delta_{p q_{4}}\right)
\end{array}\right) .
$$

To obtain the Casimir energy we need to determine the eigenfrequencies of all the normal modes of the electromagnetic field between the two periodic gratings. These eigenfrequencies can be summed up by making use of an argument principle, which states:

$$
\frac{1}{2 \pi i} \oint \phi(\omega) \frac{d}{d \omega} \ln f(\omega) d \omega=\sum \phi\left(\omega_{0}\right)-\sum \phi\left(\omega_{\infty}\right) .
$$

Here, $\omega_{0}$ are zeroes, $\omega_{\infty}$ are poles of the function $f(\omega)$ inside the contour of integration, and the degenerate eigenvalues are summed over according to their multiplicities. For the Casimir energy we have $\phi(\omega)=\hbar \omega / 2$. The equation for eigenfrequencies of the corresponding problem of classical electrodynamics is $f(\omega)=0$.

Consider first the plate-plate geometry when the two dielectric parallel slabs (slab 1: $z<0$, slab 2: $z>L)$ are separated by a vacuum gap $(0<z<L)$. In this case the TE and TM modes are not coupled. The equation for the TE eigenfrequencies is:

$$
f(\omega)=1-r_{\mathrm{TE}, \mathrm{bot}}^{(1)}\left(\boldsymbol{k}_{\perp}, \omega\right) r_{\mathrm{TE}, \mathrm{top}}^{(2)}\left(\boldsymbol{k}_{\perp}, \omega\right)=0 .
$$

Here $r_{\mathrm{TE}, \text { bot }}^{(1)}\left(\boldsymbol{k}_{\perp}, \omega\right)$ is the reflection coefficient of a plane wave moving down which reflects on the dielectric surface of the slab 1 at $z=0$, while $r_{\mathrm{TE}, \mathrm{top}}^{(2)}\left(\boldsymbol{k}_{\perp}, \omega\right)$ is the reflection coefficient of an upward moving plane wave which reflects on the dielectric surface of the slab 2 at $z=L$, and $\boldsymbol{k}_{\perp}=\left(k_{x}, k_{y}\right)$. One can deduce from the Maxwell equations that $r_{\mathrm{TE}, \text { top }}^{(2)}\left(\boldsymbol{k}_{\perp}, \omega\right)=r_{\mathrm{TE}, \mathrm{bot}}^{(2)}\left(\boldsymbol{k}_{\perp}, \omega\right) \exp \left(2 i k_{z} L\right)$ [here, $r_{\mathrm{TE}, \mathrm{bot}}^{(2)}\left(\boldsymbol{k}_{\perp}, \omega\right)$ is the reflection coefficient of a downward moving TE plane wave which reflects from the dielectric slab 2 temporarily located at the position of the slab 1, i.e. at $z<0$ ]. From (57) and the analogous equation for the TM modes one immediately obtains the Lifshitz formula by making use of the argument principle (566).

For two periodic dielectrics or gratings separated by a vacuum gap one has to consider the reflection of downward and upward moving waves from the unit cell $0<k_{x}<2 \pi / \Lambda$. Imagine that we removed the top grating in the system. Then the reflection matrix of the downward moving wave is defined as $R_{\mathrm{bot}}^{(1)}$. Imagine now that we remove the bottom grating 
in the system. Then we denote the reflection matrix of the upward moving wave as $R_{\mathrm{top}}^{(2)}$. The reflection matrices $R_{\mathrm{bot}}^{(1)}, R_{\mathrm{top}}^{(2)}$ depend on the wave vectors of the incident waves, parameters of the gratings and the mutual location of the gratings. The equation for normal modes states:

$$
R_{\mathrm{bot}}^{(1)}\left(\boldsymbol{k}_{\perp}, \omega_{i}\right) R_{\mathrm{top}}^{(2)}\left(\boldsymbol{k}_{\perp}, \omega_{i}, L, x_{0}\right) \psi_{i}=\psi_{i}
$$

where $\psi_{i}$ is an eigenvector describing the normal mode with the frequency $\omega_{i}$. Instead of Eq. (57) one obtains:

$$
\operatorname{det}\left[I-R_{\mathrm{bot}}^{(1)}\left(\boldsymbol{k}_{\perp}, \omega\right) R_{\mathrm{top}}^{(2)}\left(\boldsymbol{k}_{\perp}, \omega, L, x_{0}\right)\right]=0 .
$$

For every $k_{x}, k_{y}$ the solution of (59) yields possible eigenfrequencies $\omega_{i}$ for the solutions of Maxwell equations that should be substituted into the definition of the Casimir energy $E=\sum_{i} \hbar \omega_{i} / 2$.

Suppose that the reflection matrix $R_{\text {bot }}^{(2)}$ for the reflection from the fictitious (imaginary) grating located as in Fig. 12 is known in the coordinates $(x, z)$. Performing a change of coordinates $z=-z_{1}+L, x=x_{1}-x_{0} \quad\left(x_{0}<\Lambda\right)$ in (44), it is possible to obtain the matrix $R_{\text {top }}^{(2)}$ for the reflection of upward waves from a grating with the same profile turned upsidedown and displaced from the lower grating by $\Delta x=x_{0}, \Delta z=L$ (see Fig. 13). It follows that

$$
R_{\mathrm{top}}^{(2)}\left(\boldsymbol{k}_{\perp}, i \xi, L, x_{0}\right)=Q^{*}\left(x_{0}\right) K\left(\boldsymbol{k}_{\perp}, i \xi, L\right) R_{\mathrm{bot}}^{(2)}\left(\boldsymbol{k}_{\perp}, i \xi\right) K\left(\boldsymbol{k}_{\perp}, i \xi, L\right) Q\left(x_{0}\right),
$$

where $R_{\text {bot }}^{(2)}\left(\boldsymbol{k}_{\perp}, i \xi\right)$ is the reflection matrix of the downward moving waves from the grating in the system of coordinates $(x, z)$ depicted in Fig. 12. Here, $K\left(\boldsymbol{k}_{\perp}, i \xi, L\right)$ is the diagonal $2(2 N+1)$ matrix of the form:

$$
K\left(\boldsymbol{k}_{\perp}, i \xi, L\right)=\left(\begin{array}{cc}
G_{1} & 0 \\
0 & G_{1}
\end{array}\right)
$$

with the matrix elements $\exp \left[-L \sqrt{\xi^{2}+k_{y}^{2}+\left(k_{x}+2 \pi m / \Lambda\right)^{2}}\right](m=-N, \ldots, N)$ on the main diagonal of the matrix $G_{1}$. The lateral translation $2(2 N+1)$ diagonal matrix $Q$ is defined as follows:

$$
Q\left(x_{0}\right)=\left(\begin{array}{cc}
G_{2} & 0 \\
0 & G_{2}
\end{array}\right),
$$

with matrix elements $\exp \left(2 \pi i m x_{0} / \Lambda\right)$ on the main diagonal of the matrix $G_{2}$, where $m=$ $-N, \ldots, N$. 
The summation over the eigenfrequencies is performed by making use of the argument principle (56), which yields the Casimir energy of two parallel gratings on a unit cell of a period $\Lambda$ and unit length in the $y$ direction:

$$
E^{\mathrm{C}}\left(L, x_{0}\right)=\frac{\hbar c \Lambda}{(2 \pi)^{3}} \int_{0}^{\infty} d \xi \int_{-\infty}^{\infty} d k_{y} \int_{0}^{2 \pi / \Lambda} d k_{x} \ln \operatorname{det}\left[I-R_{\mathrm{bot}}^{(1)}\left(\boldsymbol{k}_{\perp}, i \xi\right) R_{\mathrm{top}}^{(2)}\left(\boldsymbol{k}_{\perp}, i \xi, L, x_{0}\right)\right] .
$$

Here, as was defined in Sec. II, $\varphi=2 \pi x_{0} / \Lambda$ and $x_{0}$ is a lateral displacement of the two gratings. This is an exact expression valid at zero temperature for two arbitrary parallel gratings with equal periods $\Lambda$ separated by a vacuum gap.

The Casimir free energy on a unit surface $\mathcal{F}^{\mathrm{C}}$ for the system of two gratings can be written as follows:

$$
\mathcal{F}^{\mathrm{C}}(L, \varphi)=\frac{k_{B} T}{\pi^{2}} \sum_{l=0}^{\infty} \int_{0}^{\infty} d k_{y} \int_{0}^{\pi / \Lambda} d k_{x} \ln \operatorname{det}\left[I-R_{\mathrm{bot}}^{(1)}\left(\boldsymbol{k}_{\perp}, i \xi_{l}\right) R_{\mathrm{top}}^{(2)}\left(\boldsymbol{k}_{\perp}, i \xi_{l}, L, \varphi\right)\right] .
$$

This formula is valid for an arbitrary profile and an arbitrary dielectric permittivity of each grating.

When one grating has a curvature of the sphere of the radius $R \gg \Lambda$, the lateral Casimir force in this system $F_{\text {lat }}^{\mathrm{C}}$ can be obtained by combining the use of the PFA for a sphere-plate configuration and the exact formula for the free energy of the two gratings (64):

$$
F_{\text {lat }}^{\mathrm{C}}(L, \varphi)=2 \pi R \frac{2 \pi}{\Lambda} \int_{L}^{\infty} d z^{\prime} \frac{\partial \mathcal{F}^{\mathrm{C}}\left(z^{\prime}, \varphi\right)}{\partial \varphi}
$$

For the sinusoidally corrugated sphere and plate used in our experiment $L=a+A_{1}+A_{2}$ and the lateral Casimir force is given by

$$
\begin{aligned}
F_{\text {lat }}^{\mathrm{C}}(a, \varphi)= & \frac{4 k_{B} T R}{\Lambda} \int_{a}^{\infty} d z^{\prime} \sum_{l=0}^{\infty} \frac{\partial}{\partial \varphi} \int_{0}^{\infty} d k_{y} \int_{0}^{\pi / \Lambda} d k_{x} \\
& \times \ln \operatorname{det}\left[I-R_{\mathrm{bot}}^{(1)}\left(\boldsymbol{k}_{\perp}, i \xi_{l}\right) R_{\mathrm{top}}^{(2)}\left(\boldsymbol{k}_{\perp}, i \xi_{l}, z^{\prime}+A_{1}+A_{2}, \varphi\right)\right] .
\end{aligned}
$$

Different methods were developed in grating theory starting with the pioneering work of Rayleigh [72]. For our case of sinusoidal gratings we used the so-called differential method [73]. The integration of the first-order ordinary differential equations (154) with the dielectric permittivity of the generalized plasma-like model (37) in the corrugation region of each grating was based on an explicit Runge-Kutta $(4,5)$ formula, the Dormand-Prince pair, and was performed using the Matlab package. Then the reflection matrices $R_{\text {bot }}^{(1)}$ and $R_{\text {top }}^{(2)}$ were computed. The lateral Casimir force $F_{\text {lat }}^{\mathrm{C}}$ was computed using Eq. (66) for the experimental 
parameters of the first and second set of measurement. Every Matsubara term in Eq. (66) was evaluated with a precision of $1.5 \%$ which is also the computational precision of the obtained $F_{\text {lat }}^{\mathrm{C}}$.

In Fig. [7(a-c) the exact computational results for the lateral Casimir force as a function of the phase shift between the sinusoidally corrugations on both surfaces are shown by the solid lines at separations $a=124.7,128.6$, and $149.8 \mathrm{~nm}$, respectively (the the first set of measurements with corrugation amplitude on the sphere of $13.7 \mathrm{~nm}$ ). No fitting parameters are used in the comparison As can be seen in Fig. 7)(a-c), the solid lines are in a very good agreement with the experimental data shown as dots. These lines clearly demonstrate deviations from the sinusoidal behavior which decreases with the increase of separation. Thus, both the experimental data and the exact theory confirm the prediction of Ref. [38] made using the PFA approach that the lateral Casimir force is asymmetric.

In Fig. 8 the exact computational results for the $\max \left|F_{\text {lat }}^{\mathrm{C}}\right|$ are plotted as a band between the solid lines versus the separation $a$ between the mean levels of corrugations. The width of the band takes into account the computational errors equal to $1.5 \%$ and the correction to Eq. (66) due to surface roughness. The lower solid line is obtained as the computed $\max \left|F_{\text {lat }}^{\mathrm{C}}\right|$ minus $0.015 \max \left|F_{\text {lat }}^{\mathrm{C}}\right|$. The upper solid line represents $\left(1+0.015+\eta_{\text {corr }}\right) \max \left|F_{\text {lat }}^{\mathrm{C}}\right|$, where the correction factor $\eta_{\text {corr }}$ for the first set of measurement was found in Sec. V. As can be seen in Fig. 8, the exact theory is in a very good agreement with the measurement data although it deviates significantly from the theoretical band between the two dashed lines computed using the PFA approach. Again no fitting parameters are used in the theory. This allows one to conclude that with the grating period of $\Lambda=574.7 \mathrm{~nm}$ the diffraction-type effects on the lateral Casimir force have been reliably demonstrated both experimentally and theoretically.

The results of similar computations using Eq. (66) are presented by the solid lines in Fig. 9(a-c) where the lateral Casimir force is plotted as a function of the phase shift for the second set of our mesurements at $a=134,156.5$, and $179 \mathrm{~nm}$, respectively. As before no fitting parameters are used. In spite of the fact that the experimental data of this set of measurements with deeper corrugations on the sphere are more noisy, the exact theory is clearly consistent with data and confirms the asymmetric (nonsinusoidal) character of the lateral Casimir force. In Fig. 10 the exact computational results for the $\max \left|F_{\text {lat }}^{\mathrm{C}}\right|$ in the second set of our measurements versus separation are presented as the band between the 
two solid lines. The width of this band takes into account both the computational errors and the surface roughness. It was found using the same procedure, as for the first set of measurements, with the replacement of $\eta_{\text {corr }}$ by $\tilde{\eta}_{\text {corr }}$ (see Sec. V). As in previous comparisons, no fitting parameters are used. It can be seen in Fig. 10 that in spite of the larger noise in the second set of measurements, the data are consistent with the exact theory. The deviations of both the exact theory and the measurement data from the theoretical prediction of the PFA approach are even more pronounced than in the first set of measurements. Thus, the second set of our measurements confirms the observation of the diffraction effects in the lateral Casimir force.

\section{CONCLUSIONS AND DISCUSSION}

In the foregoing we have presented the results of an experimental and theoretical investigation of the lateral Casimir force which arises between Au-coated aligned sinusoidally

corrugated surfaces of a sphere and a plate spaced in close proximity to each other. The most distinctive feature of this experiment in comparison with the one performed previously [37, 38] is the use of the grating with much smaller corrugation period which permitted to enter the region of parameters outside the applicability of the PFA and observe the nontrivial diffraction-type effects. Another distinctive feature of this experiment is the use of much deeper corrugations on both the plate and the sphere. This allowed us to observe an asymmetry of the lateral Casimir force (i.e., the deviation of its profile from perfect sine) which was predicted [38] in the framework of the PFA approach, but not observed up to date due to the use of small corrugation amplitudes. In this paper we have presented details of the experimental setup, the original procedure allowing to imprint relatively deep corrugations using a metallized template grating on a sphere of about $100 \mu \mathrm{m}$ radius, and the process of electrostatic calibrations giving the possibility to measure small forces and at short absolute separations with high precision. The dependence of the lateral Casimir force on the phase shift between the corrugations on the sphere and the plate was measured at many different separation distances in the two sets of measurements with different amplitudes of corrugations on the sphere. The maximum value of the lateral force as a function of separation was also investigated in the two sets of measurements. Both the random and systematic errors of the measured forces and separations were found and the total experimental errors were 
calculated at a $95 \%$ confidence level.

The experimental data were compared with an approximate theory using the PFA and with the exact theory based on the scattering approach. Note that exact calculation methods allowing the evaluation of the Casimir force between bodies of arbitrary shape and made of any real material have been actively investigated since 2006 [35, 36, 61 71]. However, until the present paper and the rapid communication [41] there was no detailed comparison of exact theoretical results beyond the Lifshitz theory taking into account such experimental conditions as real material properties of the test bodies, surface roughness and nonzero temperature with measurement data of any specific experiment. Using the exact theory, the computed dependences of the lateral Casimir force on the phase shift and of the maximum magnitude of the lateral force as a function of separation were found to be in agreement with the experimental data. Regarding the PFA approach, the computational results were found to be excluded by the data. This provides the quantitative confirmation for the fact that this experiment was performed outside the applicability region of the PFA and marks the beginning of investigations of diffraction effects in the phenomenon of the lateral Casimir force.

As was already noted in the Introduction, the lateral Casimir force might find applications in nanotechnology. Specifically, it was proposed to use this effect for the frictionless transmission of lateral motion by means of a nanoscale rack and pinion without intermeshing cogs [28, 29] or in a ratchet with asymmetric corrugations driven by the Casimir force [30]. The above experimental and theoretical results bring us closer to the realization of such kinds of micromachines. In future it is planned to perform similar experiments with corrugations of more complicated shape which provides an opportunity to modulate the respective lateral Casimir force.

\section{Acknowledgment}

This work was supported by the NSF Grant No. PHY0653657 (measurement of the lateral Casimir force) and DOE Grant No. DE-FG02-04ER46131 (calculation of the electric and Casimir forces). V.N.M. and U.M. were also supported by the DARPA Grant N66001-091-2069. G.L.K. and V.M.M. were partially supported by the DFG Grant GE 696/10-1. V.N.M. was partially supported by the Grants RNP 2.1.1/1575 and RFBR 07-01-00692- 
a. The authors are grateful to A. V. Boukhanovsky, I. S. Zudina and to the Institute of Computer Technologies of St.Petersburg State University of Information Technologies, Mechanics and Optics where the numerical computations were performed.

[1] H. B. G. Casimir, Proc. K. Ned. Akad. Wet. 51, 793 (1948).

[2] M. Bordag, G. L. Klimchitskaya, U. Mohideen, and V. M. Mostepanenko, Advances in the Casimir Effect (Oxford University Press, Oxford, 2009).

[3] P. W. Milonni, The Quantum Vacuum (Academic Press, San Diego, 1994).

[4] V. M. Mostepanenko and N. N. Trunov, The Casimir Effect and its Applications (Clarendon, Oxford, 1997).

[5] K. A. Milton, The Casimir Effect (World Scientific, Singapore, 2001).

[6] G. Plunien, B. Müller, and W. Greiner, Phys. Rep. 134, 87 (1986).

[7] M. Kardar and R. Golestanian, Rev. Mod. Phys. 71, 1233 (1999).

[8] M. Bordag, U. Mohideen, and V. M. Mostepanenko, Phys. Rep. 353, 1 (2001).

[9] K. A. Milton, J. Phys. A: Math. Gen. 37, R209 (2004).

[10] S. K. Lamoreaux, Rep. Progr. Phys. 68, 201 (2005).

[11] G. L. Klimchitskaya, U. Mohideen, and V. M. Mostepanenko, Rev. Mod. Phys. 81, 1827 (2009).

[12] M. Krech, The Casimir Effect in Critical Systems (World Scientific, Singapore, 1994).

[13] H. B. Chan, V. A. Aksyuk, R. N. Kleiman, D. J. Bishop, and F. Capasso, Science, 291, 1941 (2001).

[14] H. B. Chan, V. A. Aksyuk, R. N. Kleiman, D. J. Bishop, and F. Capasso, Phys. Rev. Lett. 87, 211801 (2001).

[15] E. Buks and M. L. Roukes, Phys. Rev. B 63, 033402 (2001).

[16] F. M. Serry, D. Walliser, and G. J. Maclay, J. Appl. Phys. 84, 2501 (1998).

[17] V. A. Parsegian and G. H. Weeiss, J. Colloid. Interface Sci. 40, 35 (1972).

[18] Yu. S. Barash, Izv. Vuzov., Radiofiz. 16, 1227 (1973) [Radiophys. Quantum Electron. 16, 945 (1973)].

[19] S. J. van Enk, Phys. Rev. A 52, 2569 (1995).

[20] R. Golestanian and M. Kardar, Phys. Rev. Lett. 78, 3421 (1997). 
[21] R. Golestanian and M. Kardar, Phys. Rev. A 58, 1713 (1998).

[22] T. Emig, A. Hanke, R. Golestanian, and M. Kardar, Phys. Rev. Lett. 87, 260402 (2001).

[23] T. Emig, A. Hanke, R. Golestanian, and M. Kardar, Phys. Rev. A 67, 022114 (2003).

[24] R. B. Rodrigues, P. A. Maia Neto, A. Lambrecht, and S. Reynaud, Phys. Rev. Lett. 96, $100402(2006)$.

[25] R. B. Rodrigues, P. A. Maia Neto, A. Lambrecht, and S. Reynaud, Phys. Rev. A 75, 062108 (2007).

[26] T. Emig, Europhys. Lett. 62, 466 (2003).

[27] R. Büscher and T. Emig, Phys. Rev. A 69, 062101 (2004).

[28] A. Ashourvan, M. Miri, and R. Golestanian, Phys. Rev. Lett. 98, 140801 (2007).

[29] A. Ashourvan, M. Miri, and R. Golestanian, Phys. Rev. E 75, 040103(R) (2007).

[30] T. Emig, Phys. Rev. Lett. 98, 160801 (2007).

[31] J. N. Munday, D. Iannuzzi, Y. Barash, and F. Capasso, Phys. Rev. A 71, 042102 (2005).

[32] R. B. Rodrigues, P. A. Maia Neto, A. Lambrecht, and S. Reynaud, Europhys. Lett. 76, 0822 (2006).

[33] A. Roy and U. Mohideen, Phys. Rev. Lett. 82, 4380 (1999).

[34] H. B. Chan, Y. Bao, J. Zou, R. A. Cirelli, F. Klemens, W. M. Mansfield, and C. S. Pai, Phys. Rev. Lett. 101, 030401 (2008).

[35] A. Lambrecht and V. N. Marachevsky, Phys. Rev. Lett. 101, 160403 (2008).

[36] A. Lambrecht and V. N. Marachevsky, Int. J. Mod. Phys. A 24, 1789 (2009).

[37] F. Chen, G. L. Klimchitskaya, U. Mohideen, and V. M. Mostepanenko, Phys. Rev. Lett. 88, 101801 (2002).

[38] F. Chen, G. L. Klimchitskaya, U. Mohideen, and V. M. Mostepanenko, Phys. Rev. A 66, $032113(2002)$.

[39] G. L. Klimchitskaya, U. Mohideen, and V. M. Mostepanenko, J. Phys. A: Math. Theor. 40, 13485 (2007).

[40] R. S. Decca, D. López, E. Fischbach, G. L. Klimchitskaya, D. E. Krause, and V. M. Mostepanenko, Eur. Phys. J. C 51, 963 (2007).

[41] H.-C. Chiu, G. L. Klimchitskaya, V. N. Marachevsky, V. M. Mostepanenko, and U. Mohideen, Phys. Rev. B 80, 121402(R) (2009).

[42] H.-C. Chiu, C.-C. Chang, R. Castillo-Garza, F. Chen, and U. Mohideen, J. Phys. A: Math. 
Theor. 41, 164022 (2008).

[43] F. Chen, G. L. Klimchitskaya, U. Mohideen, and V. M. Mostepanenko, Phys. Rev. A 69, 022117 (2004).

[44] W. R. Smythe, Electrostatics and Electrodynamics (McGraw-Hill, New York, 1950).

[45] F. Chen, U. Mohideen, G. L. Klimchitskaya, and V. M. Mostepanenko, Phys. Rev. A 74, $022103(2006)$.

[46] http://www.comsol.com

[47] L. Boyer, F. Houzé, A. Tonck, J.-L. Loubet, and J.-M. Georges, J. Phys. D: Appl. Phys. 27, 1504 (1994).

[48] R. S. Decca, E. Fischbach, G. L. Klimchitskaya, D. E. Krause, D. López, U. Mohideen, and V. M. Mostepanenko, arXiv:0904.4720.

[49] F. Chen, G. L. Klimchitskaya, V. M. Mostepanenko, and U. Mohideen, Phys. Rev. Lett. 97, $170402(2006)$.

[50] W. J. Kim, M. Brown-Hayes, D. A. R. Dalvit, J. H. Brownell, and R. Onofrio, Phys. Rev. A 79, 026102 (2009).

[51] F. Chen and U. Mohideen, Rev. Sci. Instrum. 72, 3100 (2001).

[52] S. G. Rabinovich, Measurement Errors and Uncertainties: Theory and Practice (Springer, New York, 2000).

[53] G. L. Klimchitskaya, F. Chen, R. S. Decca, E. Fischbach, , D. E. Krause, D. López, U. Mohideen, and V. M. Mostepanenko, J. Phys. A: Math. Gen. 39, 6485 (2006).

[54] A. Canaguier-Durand, P. A. Maia Neto, I. Cavero-Pelaez, A. Lambrecht, and S. Reynaud, Phys. Rev. Lett. 102, 230404 (2009).

[55] T. Emig, J. Stat. Mech. P04007 (2008).

[56] D. E. Krause, R. S. Decca, D. López, and E. Fischbach, Phys. Rev. Lett. 98, 050403 (2007).

[57] Handbook of Optical Constants of Solids, ed. E. D. Palik (Academic, New York, 1985).

[58] M. J. Renne, Physica 56, 125 (1971).

[59] R. Balian and B. Duplantier, Ann. Phys. (N.Y.) 104, 300 (1977).

[60] R. Balian and B. Duplantier, Ann. Phys. (N.Y.) 112, 165 (1978).

[61] T. Emig, R. L. Jaffe, M. Kardar, and A. Scardicchio, Phys. Rev. Lett. 96, 080403 (2006).

[62] T. Emig, N. Graham, R. L. Jaffe, and M. Kardar, Phys. Rev. Lett. 99, 170403 (2007).

[63] T. Emig, N. Graham, R. L. Jaffe, and M. Kardar, Phys. Rev. D 77, 025005 (2008). 
[64] O. Kenneth and I. Klich, Phys. Rev. Lett. 97, 160401 (2006).

[65] O. Kenneth and I. Klich, Phys. Rev. B 78, 014103 (2008).

[66] A. Bulgac, P. Magierski, and A. Wirzba, Phys. Rev. D 73, 025007 (2006).

[67] K. A. Milton and J. A. Wagner, J. Phys. A: Math. Theor. 41, 155402 (2008).

[68] S. J. Rahi, T. Emig, R. L. Jaffe, and M. Kardar, Phys. Rev. A 78, 012104 (2008).

[69] S. J. Rahi, T. Emig, N. Graham, R. L. Jaffe, and M. Kardar, Phys. Rev. D 80, 085021 (2009).

[70] P. A. Maia Neto, A. Lambrecht, and S. Reynaud, Phys.Rev. A 78, 012115 (2008).

[71] M. Bordag, Phys. Rev. D 73, 125018 (2006).

[72] O. M. Rayleigh, Proc. Roy. Soc. A 79, 399 (1907).

[73] Selected Papers on Diffraction Gratings, ed. D. Maystre (SPIE Press, Bellingham, 1993). 
Figures 
FIG. 1: Schematic of the experimental setup (see text for further details). Insertion shows the obstructed side of the sphere with imprinted corrugations. 
FIG. 2: (Color online) (a) An AFM scan of the grating surface showing the sinusoidal corrugations covered with stochastic roughness. (b) A typical section of the grating surface along a $y=$ const plane. The solid line shows a sine function obtained from the fit. 
FIG. 3: (Color online) (a) An AFM scan of the surface of the sphere used in the first set of measurements showing the imprinted sinusoidal corrugations covered with stochastic roughness. (b) A typical section of the grating surface along a $y=$ const plane. The solid line shows a sine function obtained from the fit. 
FIG. 4: (Color online) The imprinted corrugations on the sphere used in the first set of measurements. The lighter area shows higher points and hence demonstrates the sphericity of the imprinted surface. 
FIG. 5: (Color online) The imprinted corrugations on the sphere used in the second set of measurements. The lighter area shows higher points and hence demonstrates the sphericity of the imprinted surface. 
FIG. 6: The deflection signal due to the lateral electric force versus the phase shift (a) before and (b) after the correction to the tilt between the grating and the $x$-axis was introduced. 
FIG. 7: (Color online) The experiment (dots) and the exact theory (solid lines) for the lateral Casimir force versus the lateral displacement normalized for the corrugation period at the separations (a) $124.7 \mathrm{~nm}$, (b) $128.6 \mathrm{~nm}$, and (c) $149.8 \mathrm{~nm}$ in the first set of measurements with corrugation amplitude on the sphere of $13.7 \mathrm{~nm}$. No fitting parameters are used. 
FIG. 8: The experimental data (crosses) and theoretical values computed using the exact theory (the band between the solid lines) and using the proximity force approximation (the band between the dashed lines) for the maximum magnitudes of the lateral Casimir force versus separation in the first set of measurements. No fitting parameters are used. 
FIG. 9: (Color online) The experiment (dots) and the exact theory (solid lines) for the lateral Casimir force versus the lateral displacement normalized for the corrugation period at the separations (a) $134 \mathrm{~nm}$, (b) $156.9 \mathrm{~nm}$, and (c) $179 \mathrm{~nm}$ in the second set of measurements with corrugation amplitude on the sphere of $25.5 \mathrm{~nm}$. No fitting parameters are used. 
FIG. 10: The experimental data (crosses) and theoretical values computed using the exact theory (the band between the solid lines) and using the proximity force approximation (the band between the dashed lines) for the maximum magnitudes of the lateral Casimir force versus separation in the second set of measurements. No fitting parameters are used. 
FIG. 11: (Color online) Two longitudinal gratings of different shape and same period $\Lambda$. 
FIG. 12: (Color online) The fictitious longitudinal grating for which one evaluates the reflection $\operatorname{matrix} R_{\mathrm{bot}}^{(2)}$ (see text for further discussion). 
FIG. 13: (Color online) The top longitudinal grating in Fig. 11 for which one evaluates the reflection

matrix $R_{\text {top }}^{(2)}$. The normal and lateral shifts with respect to the fictitious grating shown in Fig. 12 are denoted by $L$ and $x_{0}$, respectively. 
Tables 
TABLE I: The mean values (column 2), the variances of the mean (column 3), the systematic errors (column 4), and the total experimental errors at a $95 \%$ confidence level (column 5) of the measured maximum magnitudes of the lateral Casimir force $f \equiv \max \left|F_{\text {lat }}^{\mathrm{C}}\right|$ at different separations (column 1) for the first set of measurements.

\begin{tabular}{ccccc}
\hline \hline $\begin{array}{c}a \\
(\mathrm{~nm})\end{array}$ & $\begin{array}{c}s_{\bar{f}} \\
(\mathrm{pN})\end{array}$ & $\begin{array}{c}\Delta^{\text {syst }} f \\
(\mathrm{pN})\end{array}$ & $\begin{array}{c}\Delta^{\text {tot }} f \\
(\mathrm{pN})\end{array}$ \\
\hline 121.1 & 3.6 & 2.4 & 11.1 \\
124.7 & 1.3 & 1.7 & 4.7 \\
128.6 & 36.4 & 2.4 & 1.3 & 7.1 \\
137.3 & 27.8 & 0.72 & 0.86 & 2.5 \\
149.8 & 0.56 & 0.48 & 1.8 \\
162.6 & 17.6 & 0.23 & 0.30 & 0.8 \\
175.3 & 9.50 & 0.37 & 0.20 & 1.1 \\
188.1 & 5.76 & 0.40 & 0.13 & 1.1 \\
\hline \hline
\end{tabular}


TABLE II: The mean values (column 2), the variances of the mean (column 3), the systematic errors (column 4), and the total experimental errors at a $95 \%$ confidence level (column 5) of the measured maximum magnitudes of the lateral Casimir force $f \equiv \max \left|F_{\text {lat }}^{\mathrm{C}}\right|$ at different separations (column 1) for the second set of measurements.

\begin{tabular}{ccccc}
\hline \hline $\begin{array}{c}a \\
(\mathrm{~nm})\end{array}$ & $\begin{array}{c}s_{\bar{f}} \\
(\mathrm{pN})\end{array}$ & $\begin{array}{c}\Delta^{\text {syst }} f \\
(\mathrm{pN})\end{array}$ & $\begin{array}{c}\Delta^{\text {tot }} f \\
(\mathrm{pN})\end{array}$ \\
\hline 133.9 & 52.5 & 4.5 & 2.5 & 12.0 \\
145.2 & 1.35 & 1.2 & 3.9 \\
156.5 & 24.1 & 0.60 & 0.67 & 1.9 \\
179.0 & 13.6 & 0.26 & 0.35 & 0.86 \\
201.6 & 6.62 & 0.24 & 0.20 & 0.69 \\
224.1 & 3.49 & 0.31 & 0.15 & 0.81 \\
246.6 & 2.37 & 0.25 & 0.10 & 0.63 \\
\hline \hline
\end{tabular}


This figure "figLL-1.gif" is available in "gif" format from: http://arxiv.org/ps/1002.3936v1 
This figure "figLL-2.gif" is available in "gif" format from: http://arxiv.org/ps/1002.3936v1 
This figure "figLL-3.gif" is available in "gif" format from: http://arxiv.org/ps/1002.3936v1 
This figure "figLL-4.gif" is available in "gif" format from: http://arxiv.org/ps/1002.3936v1 
This figure "figLL-5.gif" is available in "gif" format from: http://arxiv.org/ps/1002.3936v1 
This figure "figLL-6.gif" is available in "gif" format from: http://arxiv.org/ps/1002.3936v1 
This figure "figLL-7.gif" is available in "gif" format from: http://arxiv.org/ps/1002.3936v1 
This figure "figLL-8.gif" is available in "gif" format from: http://arxiv.org/ps/1002.3936v1 
This figure "figLL-9.gif" is available in "gif" format from: http://arxiv.org/ps/1002.3936v1 
This figure "figLL-10.gif" is available in "gif" format from: http://arxiv.org/ps/1002.3936v1 
This figure "figLL-11.gif" is available in "gif" format from: http://arxiv.org/ps/1002.3936v1 
This figure "figLL-12.gif" is available in "gif" format from: http://arxiv.org/ps/1002.3936v1 
This figure "figLL-13.gif" is available in "gif" format from: http://arxiv.org/ps/1002.3936v1 